\title{
Performance Comparison of Medium Access Control Schemes for IEEE 802.11
}

\author{
Thesis Supervisor: Hasan Shahid Ferdous
}

\section{Submitted by:}

$\begin{array}{ll}\text { Lubna Ahmed } & 09221050 \\ \text { Mahjabeen Alam } & 09221121 \\ \text { Amran Kabir } & 09221133\end{array}$

A dissertation submitted in fulfillment of the requirements for the degree of BSc. in Electrical and Electronics Engineering

April 2012 


\section{Contents}

List of Figures

List of Tables

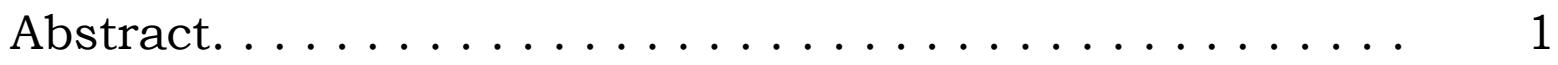

1 Introduction. . . . . . . . . . . . . . . . . . 1

2 Performance Metrics. .................. 3

3 Related Work ....................... 4

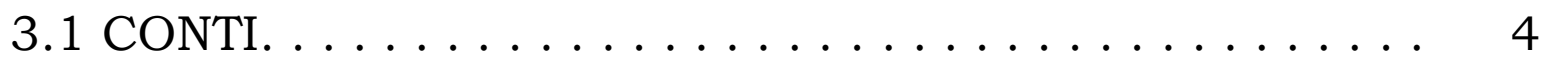

3.2 Example ......................... 4

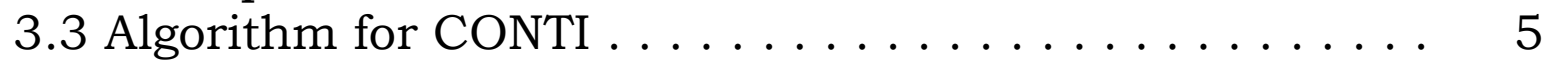

3.4 PREMA ........................ 6

3.5 Example. ...................... 6

3.6 Algorithm for PREMA . . . . . . . . . . . . . . 7

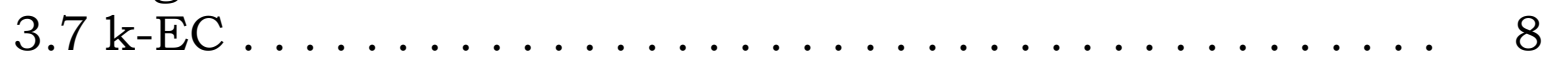

3.8 Example ....................... 9

3.9 Algorithm for k-EC. . . . . . . . . . . . . . 9

4 Simulation Results(Saturated Model). . . . . . . . . . . . . . . 10

4.1 Parameters. . . . . . . . . . . . . . . . . . . 10

4.2 Percentage Collision. . . . . . . . . . . . . . . . . 12

4.3 Throughput. . . . . . . . . . . . . . . . . . 13

4.4 Average time wasted in contention resolution. . . . . . . . 15

4.5 Delay. . . . . . . . . . . . . . . . . . . . . . . . . 17

4.6 Summary of Observations. . . . . . . . . . . . 18

5 The Unsaturated Model. . . . . . . . . . . . . . . . . . 18

5.1 Simulation Results. . . . . . . . . . . . . . . . . . 19

5.2 Mean Offtime $=100 \mu \mathrm{s} \ldots \ldots \ldots \ldots \ldots \ldots$

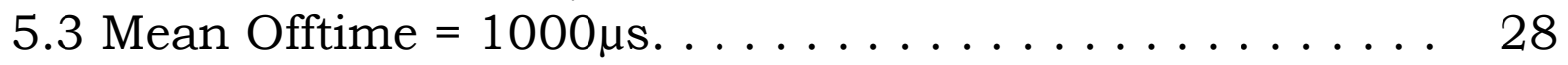

5.4 Mean Offtime $=10000 \mu$ s. . . . . . . . . . . . . 35

5.5 Summary of Observations. . . . . . . . . . . . . 43

References. .......................... 44 


\section{$\underline{\text { List of Figures }}$}

Fig 3.1: Contention resolution using CONTI

Fig 3.2: PREMA scenario with six nodes

Fig 3.3: The time event sequencing of k-EC

Fig 3.4: An example of a 6-EC

Fig 4.1: Plot for percentage collision comparison for DCF, CONTI, PREMA and $\mathrm{k}$-EC from 1 to 50 nodes

Fig 4.2: Plot for throughput for DCF, CONTI, PREMA and k-EC from 1 to 50 nodes

Fig 4.3: Bar chart for comparison of throughput for 10, 20, 30, 40 and 50 nodes for DCF, CONTI, PREMA and k-EC

Fig 4.4: Plot for average time wasted in contention for DCF, CONTI, PREMA and $\mathrm{k}$-EC from 1 to 50 nodes

Fig 4.5: Bar chart for comparison of average time wasted in contention for 10, 20, 30, 40 and 50 nodes for DCF, CONTI, PREMA and k-EC

Fig 4.6: Plot for average delay for DCF, CONTI, PREMA and k-EC from 1 to 50 nodes

Fig 4.7: Bar chart for comparison of average delay for 10, 20, 30, 40 and 50 nodes for DCF, CONTI, PREMA and k-EC

Fig 5.1: Plot for collision rate with mean offtime $=100 \mu$ s for DCF, CONTI, PREMA and k-EC from 1 to 50 nodes

Fig 5.2: Plot for throughput with mean offtime $=100 \mu$ s for DCF, CONTI, PREMA and $\mathrm{k}$-EC from 1 to 50 nodes

Fig 5.3: Bar chart for comparison of throughput for 10, 20, 30, 40 and 50 nodes for DCF, CONTI, PREMA and k-EC with mean offtime $=100 \mu \mathrm{s}$

Fig 5.4: Plot for delay comparison with mean offtime $=100 \mu$ s for DCF, CONTI, PREMA and k-EC from 1 to 50 nodes

Fig 5.5: Bar chart for delay comparison for 10, 20, 30, 40 and 50 nodes for DCF, CONTI, PREMA and k-EC with mean offtime $=100 \mu \mathrm{s}$ 
Fig 5.6: Plot for average time wasted in contention with mean offtime $=100 \mu$ s for DCF, CONTI, PREMA and k-EC from 1 to 50 nodes

Fig 5.7: Bar chart for average time wasted in contention for 10, 20, 30, 40 and 50 nodes for DCF, CONTI, PREMA and $\mathrm{k}-\mathrm{EC}$ with mean offtime $=100 \mu \mathrm{s}$

Fig 5.8: Plot for queuing delay with mean offtime $=100 \mu$ s for DCF, CONTI, PREMA and $\mathrm{k}-\mathrm{EC}$ from 1 to 50 nodes

Fig 5.9: Bar chart for queuing delay for 10, 20, 30, 40 and 50 nodes for DCF, CONTI, PREMA and k-EC with mean offtime $=100 \mu \mathrm{s}$

Fig 5.10: Plot for collision rate with mean offtime $=1000 \mu$ s for DCF, CONTI, PREMA and k-EC from 1 to 50 nodes

Fig 5.11: Plot for throughput with mean offtime $=1000 \mu \mathrm{s}$ for DCF, CONTI, PREMA and k-EC from 1 to 50 nodes

Fig 5.12: Bar chart for comparison of throughput for 10, 20, 30, 40 and 50 nodes for DCF, CONTI, PREMA and k-EC with mean offtime $=1000 \mu \mathrm{s}$

Fig 5.13: Plot for average delay with mean offtime $=1000 \mu$ s for DCF, CONTI, PREMA and $\mathrm{k}-\mathrm{EC}$ from 1 to 50 nodes

Fig 5.14: Bar chart for comparison of average delay for 10, 20, 30, 40 and 50 nodes for DCF, CONTI, PREMA and k-EC with mean offtime $=1000 \mu \mathrm{s}$

Fig 5.15: Plot for average time wasted in contention with mean offtime $=1000 \mu \mathrm{s}$ for DCF, CONTI, PREMA and k-EC from 1 to 50 nodes

Fig 5.16: Bar chart for average time wasted in contention for 10, 20, 30, 40 and 50 nodes for DCF, CONTI, PREMA and k-EC with mean offtime $=1000 \mu \mathrm{s}$

Fig 5.17: Plot for queuing delay with mean offtime $=1000 \mu$ s for DCF, CONTI, PREMA and k-EC from 1 to 50 nodes

Fig 5.18: Bar chart for queuing delay for 10, 20, 30, 40 and 50 nodes for DCF, CONTI, PREMA and $\mathrm{k}-\mathrm{EC}$ with mean offtime $=1000 \mu \mathrm{s}$

Fig 5.19: Plot for collision rate with mean offtime $=10,000 \mu$ s for DCF, CONTI, PREMA and k-EC from 1 to 50 nodes

Fig 5.20: Plot for throughput with mean offtime $=10000 \mu$ s for DCF, CONTI, PREMA and k-EC from 1 to 50 nodes 
Fig 5.21: Bar chart for comparison of throughput for 10, 20, 30, 40 and 50 nodes for DCF, CONTI, PREMA and k-EC with mean offtime $=10000 \mu \mathrm{s}$

Fig 5.22: Plot for average delay with mean offtime $=10000 \mu$ s for DCF, CONTI, PREMA and k-EC from 1 to 50 nodes

Fig 5.23: Bar chart for comparison of average delay for 10, 20, 30, 40 and 50 nodes for DCF, CONTI, PREMA and k-EC with mean offtime $=10000 \mu \mathrm{s}$

Fig 5.24: Plot for average time wasted in contention with mean offtime $=10000 \mu \mathrm{s}$ for DCF, CONTI, PREMA and k-EC from 1 to 50 nodes

Fig 5.25: Bar chart for average time wasted in contention for 10, 20, 30, 40 and 50 nodes for DCF, CONTI, PREMA and k-EC with mean offtime $=10000 \mu \mathrm{s}$

Fig 5.26: Plot for queuing delay with mean offtime $=10000 \mu$ s for DCF, CONTI, PREMA and k-EC from 1 to 50 nodes

Fig 5.27: Bar chart for queuing delay for 10, 20, 30, 40 and 50 nodes for DCF, CONTI, PREMA and k-EC with mean offtime $=10000 \mu \mathrm{s}$

\section{List of Tables}

Table 4.1: IEEE 802.11a/g OFDM PHY parameter set

Table 4.2: Parameters for CONTI, PREMA and k-EC 


\begin{abstract}
IEEE 802.11 specifies a set of protocols for a wireless LAN defined by IEEE which covers the physical and data link layer. Nowadays, IEEE 802.11 based WLAN has a widespread and ubiquitous use in providing wireless connectivity to several electronic devices like cell phones, laptops, gaming devices and so on. All these devices operate on the same medium and contend with each other in order to access the medium. All devices using the IEEE 802.11 Standard adopt a basic MAC scheme called the distributed coordination function (DCF) whose key function is contention resolution. The Binary Exponential Backoff (BEB) scheme is used to access the medium and deliver packets over a wireless network. However, the performance and efficiency of IEEE 802.11 DCF scheme is a matter of question as the number of contending stations are increasing everyday.

In this paper we make a detailed study of the performance comparison between DCF, CONTI, kEC and PREMA, which are various contention resolution schemes proposed in various independent researches. The criteria for performance comparison that we will use are collision rate, throughput and average delay between successful transmissions. Also, in this paper we are going to propose an unsaturated model for the contention schemes whose implementation and performance is consistent with the saturated mode used in the above contention schemes. Furthermore, we compare the performance for each of the contention resolution schemes in the unsaturated mode.
\end{abstract}

\title{
1 Introduction
}

Within the last few years the world has encountered a huge revolution in technology that has made access to the internet mobile and more convenient and it is none other than the Wi-Fi revolution. It is the wireless technology built on the 802.11 standard that allows wireless-enabled devices to connect to a network without wires. Wireless access today has become a necessity in our daily lives. Thus, majority of computers and other mobile devices sold to consumers today come pre-equipped with the Wi-Fi functionality.

Adopted in the year 1997, IEEE 802.11 was the first wireless LAN standard. It provides transmission in the $2.4 \mathrm{GHz} / 5 \mathrm{GHz}$ band. The IEEE has issued a series of WLAN standards, such as $802.11 \mathrm{a}, 802.11 \mathrm{~b}, 802.11 \mathrm{~g}$, and $802.11 \mathrm{n}$, and thus has made a great contribution to the wide spread use of WLAN technology. The IEEE 802.11 series adopted a common MAC protocol that provided medium access schemes. The basic scheme was called Distributed Coordination Function (DCF) which is a carrier sense multiple access protocol with collision avoidance, CSMA/CA with binary exponential backoff. In the basic access mechanism, a device that wants 
to transmit a data packet waits until the channel is sensed free for an interval named distributed inter frame spacing (DIFS). However, if two stations detect the channel as free at the same time, a collision occurs. The 802.11 thus defines this Collision Avoidance (CA) basic mechanism that reduces the probability of such collisions. To do so, before transmitting, the device has to keep on sensing the channel for an additional random time after detecting the channel as being idle for a DIFS period. This period is determined by the binary exponential backoff algorithm. The binary exponential backoff mechanism chooses a random number from a uniform distribution in the range $(0, \mathrm{w}-1)$, where $\mathrm{w}$ is called the contention window size. At the first attempt to transmit a packet, $\mathrm{w}$ is set to the minimum contention window size, $\mathrm{CW}_{\min }$ and with every attempt to transmit that is deferred, the size of $\mathrm{w}$ doubles until a maximum size is reached for the range, $\mathrm{CW}_{\max }=2 \mathrm{CW}_{\text {min }}$, where $\mathrm{m}$ is the maximum retry stage. When a packet is successfully transmitted, $w$ is reset to $\mathrm{CW}_{\text {min. }}$ Once the backoff value is chosen, MAC will decrement it each time the medium is detected to be idle for an interval of one slot time. If any other node captures the channel during this backoff period, it halts its backoff counter and waits for the channel to remain free for another DIFS period. Otherwise, it transmits its data packet when its backoff counter reaches zero. Collision occurs if two devices select the same slot to transmit data and in such cases the corresponding retry counter increments and backoff interval increases. And if the data has been successfully transmitted, the receiving station waits for a short inter-frame spacing time (SIFS) and then transmits an acknowledgement (ACK) message to the sender to confirm about the successful transmission.

There have been many researches to improve the performance of these standards and many new schemes have been proposed. For our thesis, we did extensive study of the 802.11 DCF mechanism and other protocols. These are PREMA, CONTI and k-EC. In this paper we draw a comparison between these four different access mechanisms. The comparison is based on few criterions which are delay, throughput and collision. To make a fair comparison among the schemes we use the physical layer parameters of the 802.11 a standard. 


\section{Performance metrics}

In this section, we define the parameters based on which, we evaluate the performance and efficiency of the various contention resolution schemes in the saturated and unsaturated model. We draw a comparison between these schemes and we do this based on the following criteria: Collision rate, Throughput and Average Delay.

(a)Collision Rate - When two nodes/stations try to transmit data packets over the same network at the same time, their packets collide and are lost or destroyed. This is known as collision. Collision rate is defined as the ratio of collisions to total transmissions. An increased rate of collision means higher number of retransmissions and inefficient use of channel bandwidth. Every mechanism aims to reduce the probability of collision during transmission.

\section{Percentage Collision $=$ Total no. of sent RTS - Total no. of received CTS}

\section{Total no. of sent RTS}

(b)Throughput - This parameter indicates the average rate of successful packet transmission over a channel. In other words it is the percentage of time that is actually expended in sending data bits. In our thesis, we use the following formula to calculate throughput.

\section{Normalized Throughput $=$ No. of received CTS $* 256$}

\section{Total Simulation Time}

We use the value of 256 microseconds because this is the amount of time required to send a data packet under the 802.11a physical layer parameters.

(c) Delay - Delay is defined as the time spent from when the frame arrives at a node's head of queue to the time when it is transmitted successfully. As the number of contending stations increase, it leads to less frequent transmission from the stations and thus the delay increases significantly.

\section{Average Delay $=\quad$ Total Ticks Waited \\ Total no. of received CTS}




\section{Related Work}

Numerous contention resolution schemes were proposed over time. In our paper, we compare the performance of these schemes in the saturated and unsaturated mode based on certain parameters. We mainly focus on Contention based schemes and Jamming based schemes.

\subsection{CONTI}

This MAC scheme attempts to resolve contention in a CONSTANT TIME, and thus it is named CONTI. It is a jamming based scheme that works based on the following parameters:

$\mathrm{n} \rightarrow$ number of stations contenting

$\mathrm{k} \rightarrow$ number of slots

$\mathrm{p} \rightarrow$ probability vector used by stations to decide whether to transmit a pulse or listen

Before a contention slot, a station must choose a signal based on a probability chosen from a probablity vector with length $\mathrm{k}$. The probability of choosing a signal 1 (i.e. transmit a pulse) is $p_{i}$ and that of choosing a signal 0 (i.e. listen) is $1-\mathrm{p}_{\mathrm{i}}$. The $\mathrm{i}^{\text {th }}$ element of the probability vector corresponds to $\mathrm{p}_{\mathrm{i}}$. All the nodes use the same probability vector. $n$ stations each choose a signal based on this probability and contend over k slots. During a contention slot, a station with signal 1 transmits a jam which is only a burst of energy and does not contain the actual data. A station with signal 0 listens to the channel. If it hears a jam, the station is eliminated from the contention. However, if it does not hear the presence of a jam, it stays in the contention and moves on to the next slot. At the last slot, the station that remains transmits its data frame. If there is more than one station their frames collide. In order to increase the efficiency of the scheme, the values of the parameters should be optimized.

\subsection{Example}

The figure below shows an example of this scheme. There are six contending stations. At the beginning of the contention, stations 2,4 and 5 choose a signal 1 and thus preempt stations 1,3 and 6 who choose a signal 0 . In the second slot, these remaining stations choose signal 0 and thus they all listen to the channel. No one is preempted. The stations move to the third slot where station 5 is preempted by stations 2 and 4 . In the last slot, station 2 preempts station 4 and transmits it data frame successfully. 


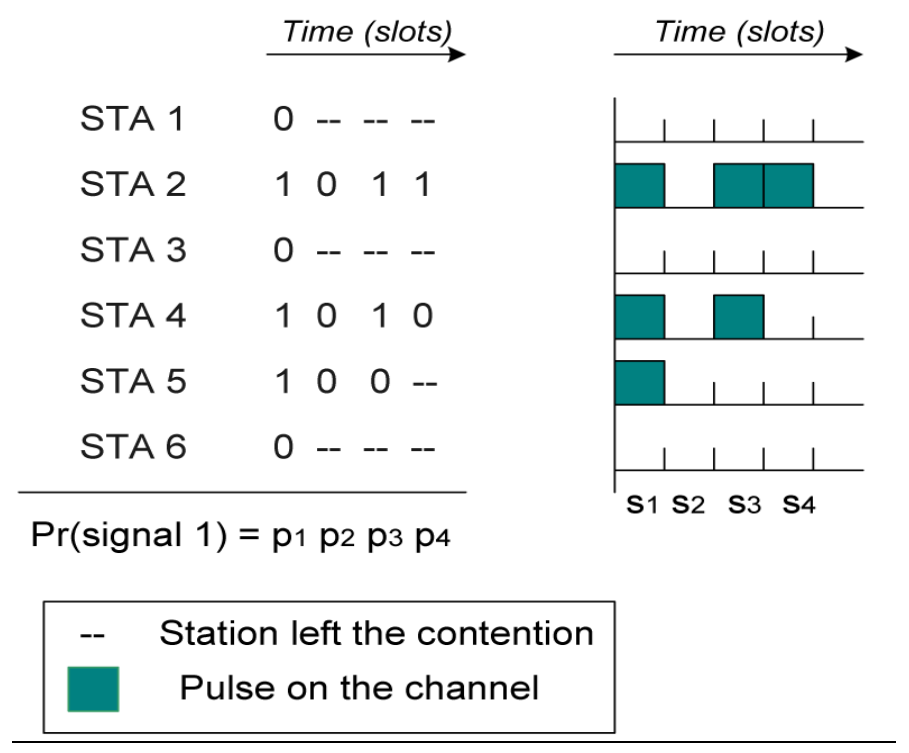

Fig 3.1: Contention resolution using CONTI

\subsection{Algorithm}

(1) Sense channel idle for DIFS;

(2) retire $=0$;

(3) $\quad \mathrm{i}=0$;

(4) while $(\mathrm{i}<\mathrm{k})$ do

(5) $\quad$ if retire $=1$;

(6) defer $\left(\mathrm{t}_{\text {slot }}\right)$

(7) $\quad$ else if retire $=0$;

(8) $\quad$ proba $=$ Sim_uniform_distribution $(0,1)$;

(9) $\quad$ if proba $<p_{i}$;

(10) $\quad$ signal $=1$;

(11) $\quad$ else signal $=0$;

(12) $\quad$ if signal $=1$;

(13) pulse $\left(t_{\text {slot }}\right)$ 


$$
\begin{aligned}
& \text { else if signal }=0 \\
& \text { listen }\left(\mathrm{t}_{\text {slot }}\right) \\
& \text { if pulse Detected }\left(\mathrm{t}_{\text {slot }}\right)=\text { true } \\
& \text { retire }=1 ; \\
& \mathrm{i}=\mathrm{i}+1 ;
\end{aligned}
$$

\subsection{PREMA}

Another contention resolution scheme goes by the name Prioritized Repeated Eliminations Multiple Access (PREMA), which is once again a jamming based protocol. All the stations contend to access a shared channel and this channel is slotted. In each slot, a station can perform one of two actions. It can either perform carrier sensing or it can send a burst onto the slot. Which action will be performed by a station depends on a probability. When, for a slot $i$, the probability is $P\left(A_{i}=t x\right)=q_{i}$, the station transmits a short burst on to the channel. If the probability is $P\left(A_{i}=c s\right)=1-q_{i}=p_{i}$, the station performs carrier sensing. If the station senses the channel to be busy, it is eliminated. If it senses the channel to be idle, it increases its idleslots counter by one. PREMA describes a parameter $h$, and when idleslots $=h$, the station has successfully eliminated all other stations and thus transmits its data frame. Since we are making a comparison between the saturated models, we ignore the probabilities mentioned above and assume that all nodes want to send a packet. Each node will transmit a burst with length sampled from a geometric distribution with parameter ' $q$ ' followed by a carrier sense slot.

\subsection{Example}

An example is given below to illustrate how this mechanism works. In this example, $\mathrm{n}=6, \mathrm{~h}=4$ and $q=0.5$. At the beginning all the stations wait for $\mathrm{T}_{\text {ifs. }}$ After that they either perform carrier sensing or transmits a burst depending on the probability. In the example $S_{2}$ and $S_{5}$ performs carrier sensing and leaves the contention as it finds the channel to be busy. In the next slots, $S_{3}$ and $S_{6}$ are eliminated in the same way. Only $S_{1}$ and $S_{4}$ survive the first elimination and moves to the second elimination round where $S_{1}$ is eliminated. $S_{4}$ performs two more eliminations and when its idleslots $=\mathrm{h}$, it transmits. 
Fig 3.2: PREMA

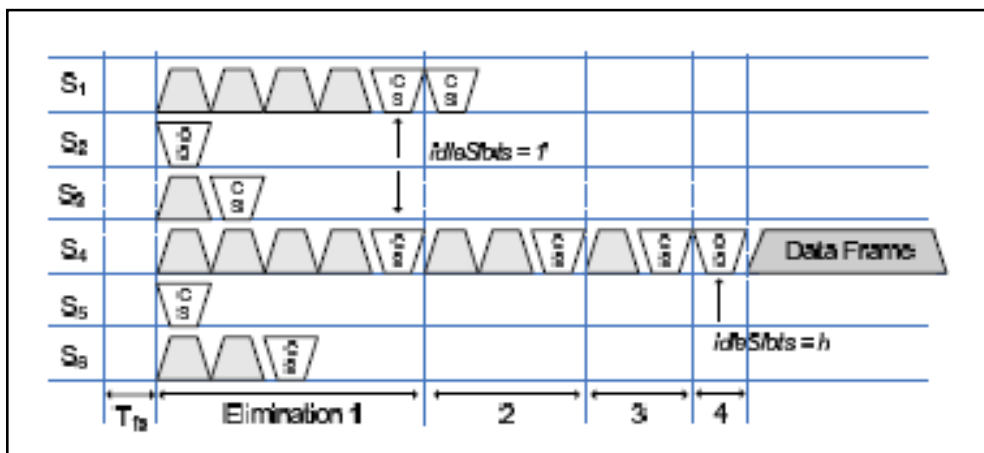

scenario with six

nodes

\subsection{Algorithm}

(1) Start

(2) Sense channel idle for $T_{\text {ifs }}$

(3) idleSlots $=0$

(4) jamLength $=$ Sim_geometric_distribution $(0, \mathrm{~m})$

(5) while idleSlots $<\mathrm{h}$ do

(6) TRANSMITNOISE $(\tau \times$ jamLength $)$

(7) $\quad$ listen $(\tau)$

(8) if pulseDetected $(\tau)=$ true

(9) goto start

(10) else

(11) $\quad$ idleSlots $=$ idleSlots +1

(12) end while

(13) TRANSMITMESSAGE $\left(\mathrm{T}_{\mathrm{m}}\right)$ 


\section{$3.7 \underline{\mathrm{k}-\mathrm{EC}}$}

The k-round Elimination Contention scheme is, like PREMA, a jamming based scheme. The basic idea of this contention resolution algorithm is to quickly reduce the number of contending nodes through elimination.

This scheme describes a parameter $\mathrm{k}$, which denotes the number of rounds of elimination and another parameter $\mathrm{m}$, which is the maximum number of slots. All the stations that are contending choose a random number uniformly in the range [0 to $\mathrm{m}-1]$ and transmit only one jam in that slot number. For example, if a station chooses 0 , it means that it sends a jam in the first slot. The station that is not jamming is listening to the channel by carrier sensing. If this station hears a jam it is out of the contention i.e. eliminated. The other stations move to the next slot.

This scheme is insensitive of the number of stations that are contending to access the channel. Thus it is very effective for use in large networks. As K-EC does not follow any backoff mechanism there is no question of unfairness. Optimized values of $\mathrm{k}$ and $\mathrm{m}$ are used. The authors use $\mathrm{k}=7$ and $\mathrm{m}=3$ as best parameters.

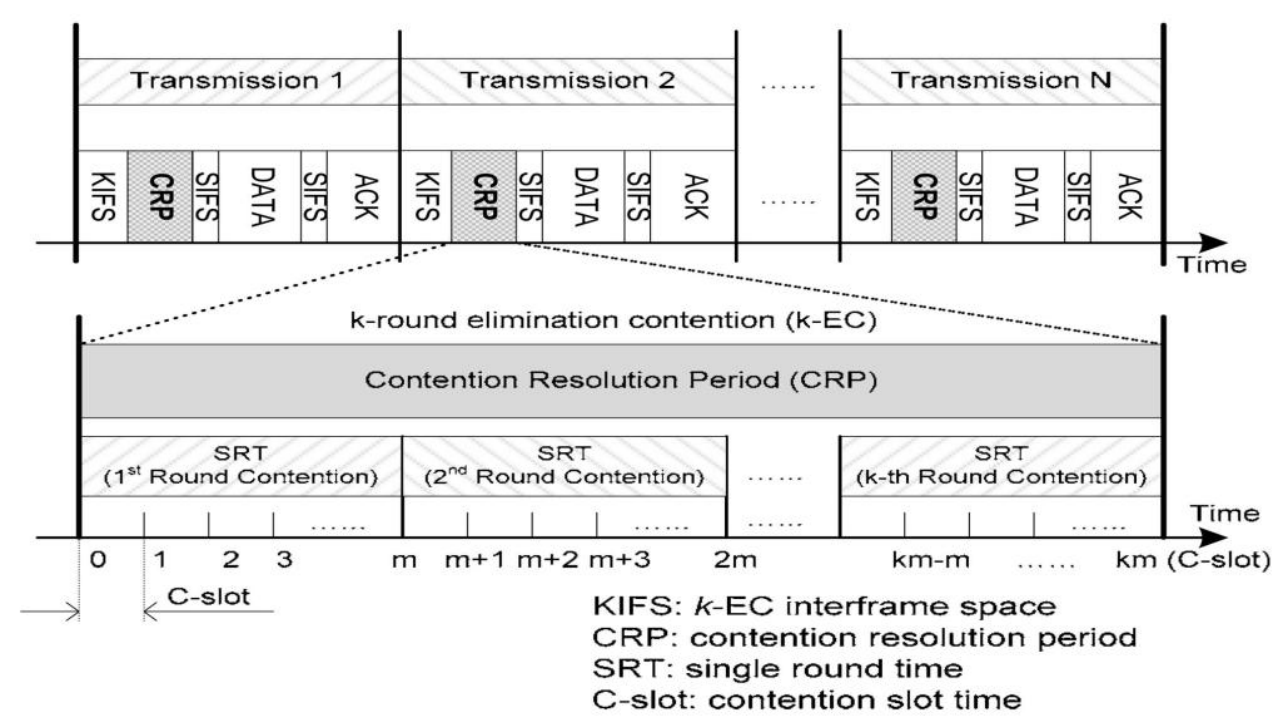

Fig 3.3: The time event sequencing of k-EC 


\subsection{Example}

The diagram below illustrates an example of $\mathrm{k}$-EC where $\mathrm{k}=6$. The black bar denotes the time when the medium is jammed.

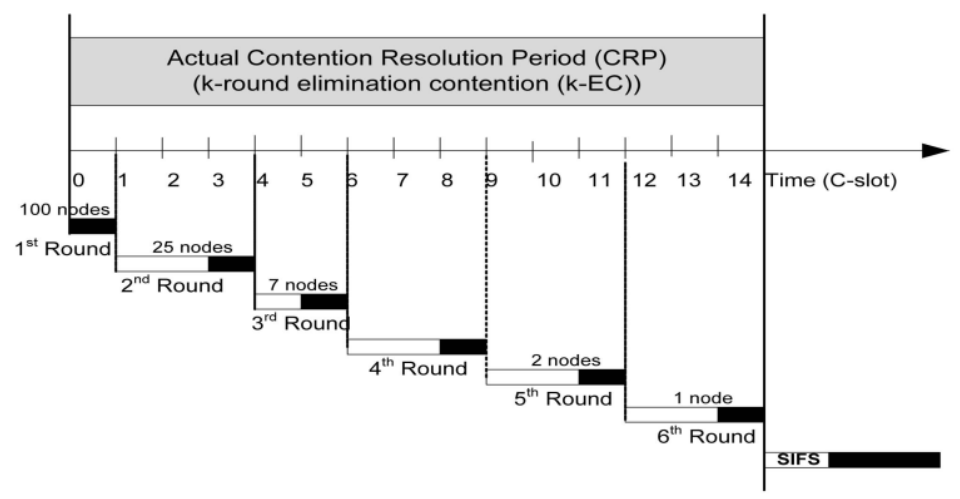

Fig 3.4: An example of a 6-EC

\subsection{Algorithm}

(1) Start

(2) Sense channel idle for KIFS

(3) no-of-rounds $=0$

(4) while no-of-rounds < k do

(5) $\quad$ slotSelect $=$ Sim_uniform_distribution $(0, \mathrm{~m}-1)$

(6) while slotSelect $\geq 0$ do

(7) $\quad$ if slotSelect $=0$

TRANSMITNOISE $\left(\mathrm{t}_{\text {slot }}\right)$

else listen $\left(t_{\text {slot }}\right)$

if pulseDetected $\left(\mathrm{t}_{\text {slot }}\right)=$ true;

goto start 


$$
\text { slotSelect }=\text { slotSelect }-1 \text {; }
$$

$$
\text { end while }
$$$$
\text { no-of-rounds }=\text { no-of-rounds }+1 \text {; }
$$

end while

TRANSMITMESSAGE $\left(\mathrm{T}_{\mathrm{m}}\right)$

\section{Simulation Results(Saturated Model)}

In this section we present the simulation results. We compare the performances of DCF, CONTI, PREMA and k-EC based on our simulation results. In order to carry out the simulations for the performance analysis of the schemes, we use the SimJava simulation environment. SimJava is a discrete event simulation library for java.

\subsection{Parameters}

The physical layer we consider is the $802.11 \mathrm{a} / \mathrm{g}$. Its parameters are summarized in the following table.

Table 2.4: IEEE 802.11 a/g OFDM PHY parameter set

\begin{tabular}{ll}
\hline Parameter & Value \\
\hline SIFS & $10 \mu \mathrm{s}$ \\
DIFS & $28 \mu \mathrm{s}$ \\
EIFS & $37 \mu \mathrm{s}$ \\
$C W_{\min }$ & 32 \\
$C W_{\max }$ & 1024 \\
Slot Time $(\sigma)$ & $9 \mu \mathrm{s}$ \\
Basic Bit Rate (BBR) & $6 \mathrm{Mbps}$ \\
Maximum Bit Rate $(\mathrm{MBR})$ & $54 \mathrm{Mbps}$ \\
MACHeader & $272 \mathrm{bits}$ \\
$T_{P C L P H e a d e r}$ & $4 \mu \mathrm{s}$ \\
$T_{P C L P P r e a m b l e}$ & $16 \mu \mathrm{s}$ \\
Symbol Duration $(\gamma)$ & $4 \mu \mathrm{s}$ \\
Maximum Retry Stage $(m)$ & 5 \\
Propagation Delay $(\delta)$ & $1 \mu \mathrm{s}$ \\
\hline
\end{tabular}

Table 4.1: IEEE 802.11a/g OFDM PHY parameter set 
In our simulations we are going to use the optimized parameters for each of the contention resolution schemes as listed in the following table.

\begin{tabular}{|l|}
\hline \multicolumn{1}{|c|}{ CONTI } \\
\hline Number of slots: $k=7$ \\
\hline Probabilities: $p=\{0.18,0.31,0.40,0.48,0.48,0.49,0.49\}$ \\
\hline \hline \multicolumn{2}{c|}{ PREMA } \\
\hline Number of eliminations: $h=4$ \\
\hline Geometric distribution parameter: $q=0.5$ \\
\hline \hline \multicolumn{2}{|c|}{ k-E } \\
\hline Number of rounds: $k=7$ \\
\hline Maximum length of a round: $m=3$ \\
\hline
\end{tabular}

Table 4.2: Parameters for CONTI, PREMA and k-EC

\subsection{Percentage Collision}

This part shows the percentage collision of the schemes. The results are shown in the following plot. 


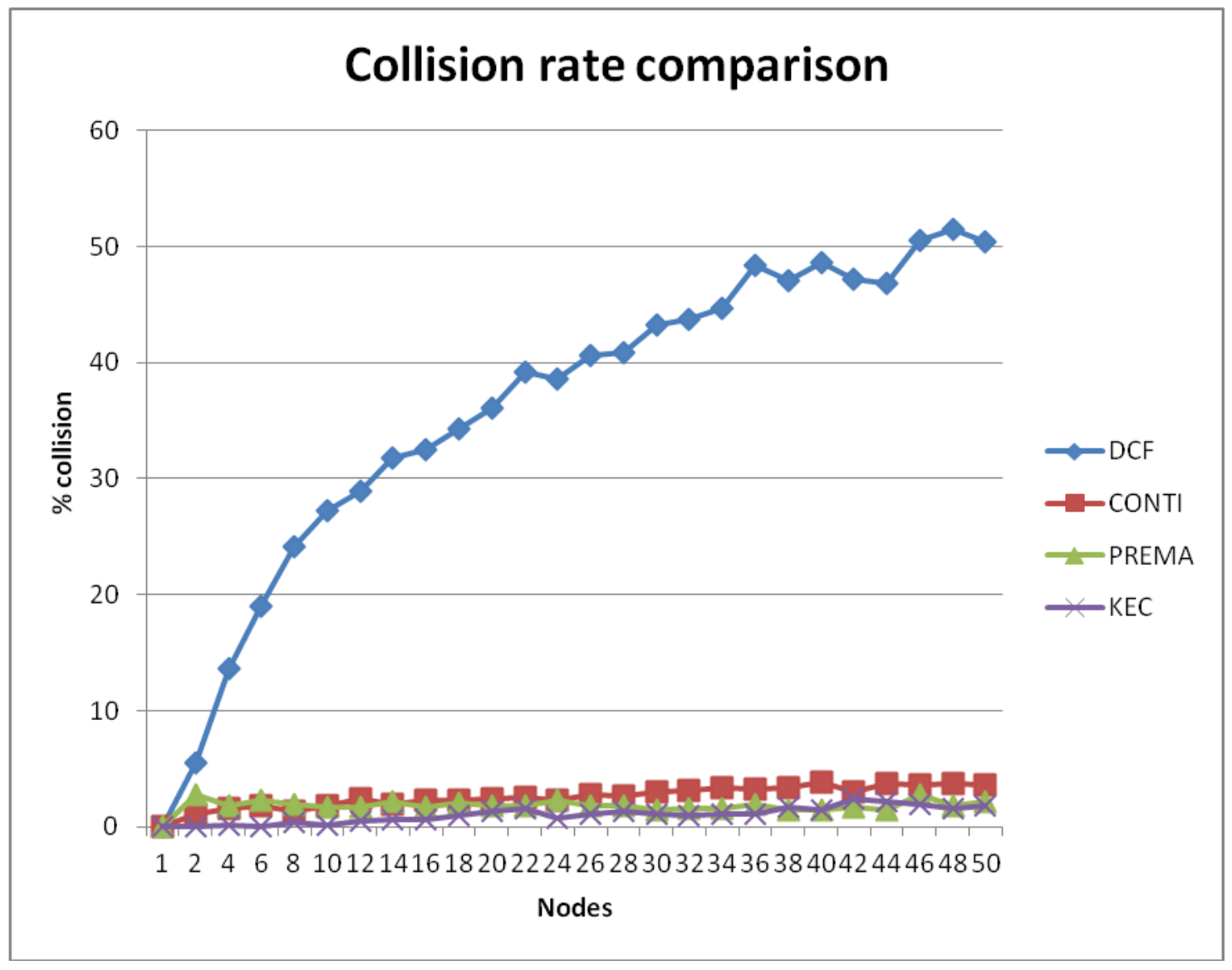

Fig 4.1: Plot for percentage collision comparison for DCF, CONTI, PREMA and k-EC from 1 to 50 nodes

The number of stations varies from 1 to 50 and the results were taken for even number of nodes. We notice that the percentage collision is lowest for PREMA and k-EC. For PREMA, percentage collision varies between 0 to 2.14 percent and for $\mathrm{k}-\mathrm{EC}$, this value varies between 0 to 1.82 . CONTI has a percentage collision that starts from 1 percent for a single node and goes up to 3.55 percent for 50 nodes. Finally, DCF had the highest value for percentage collision that was between 0 to 50.42 percent.

Finally, we draw an observation that in terms of percentage collisions, PREMA and k-EC are better contention resolution schemes compared to DCF and CONTI.

\subsection{Throughput}


Above all the other parameters, we need to understand the effect of increasing number of nodes on the normalized throughput for each of the contention resolution schemes. From the plots that follow, we can see that CONTI has the highest throughput although all the schemes have almost identical performances until up to 10 nodes.

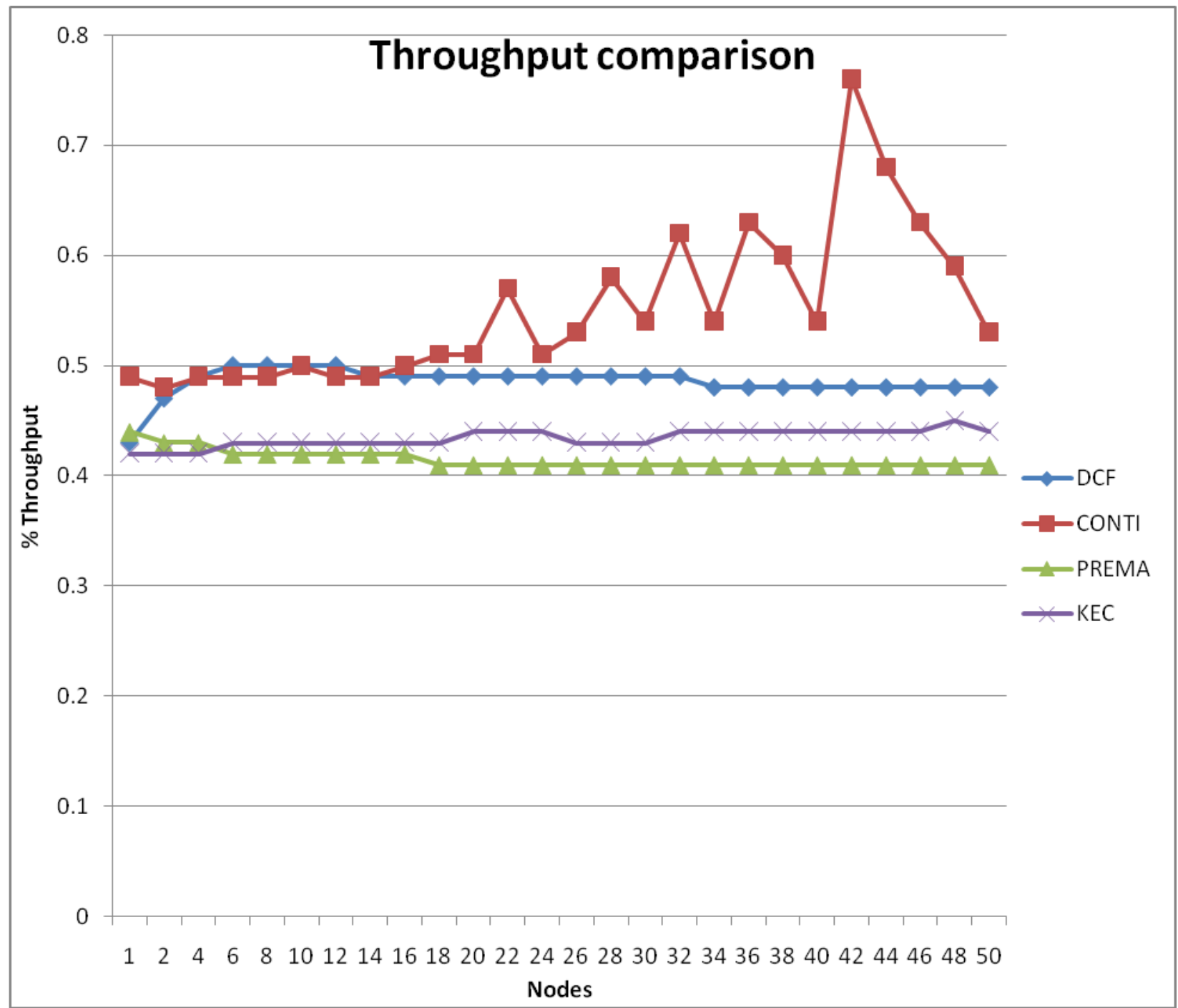

Fig 4.2: Plot for throughput for DCF, CONTI, PREMA and k-EC from 1 to 50 nodes 


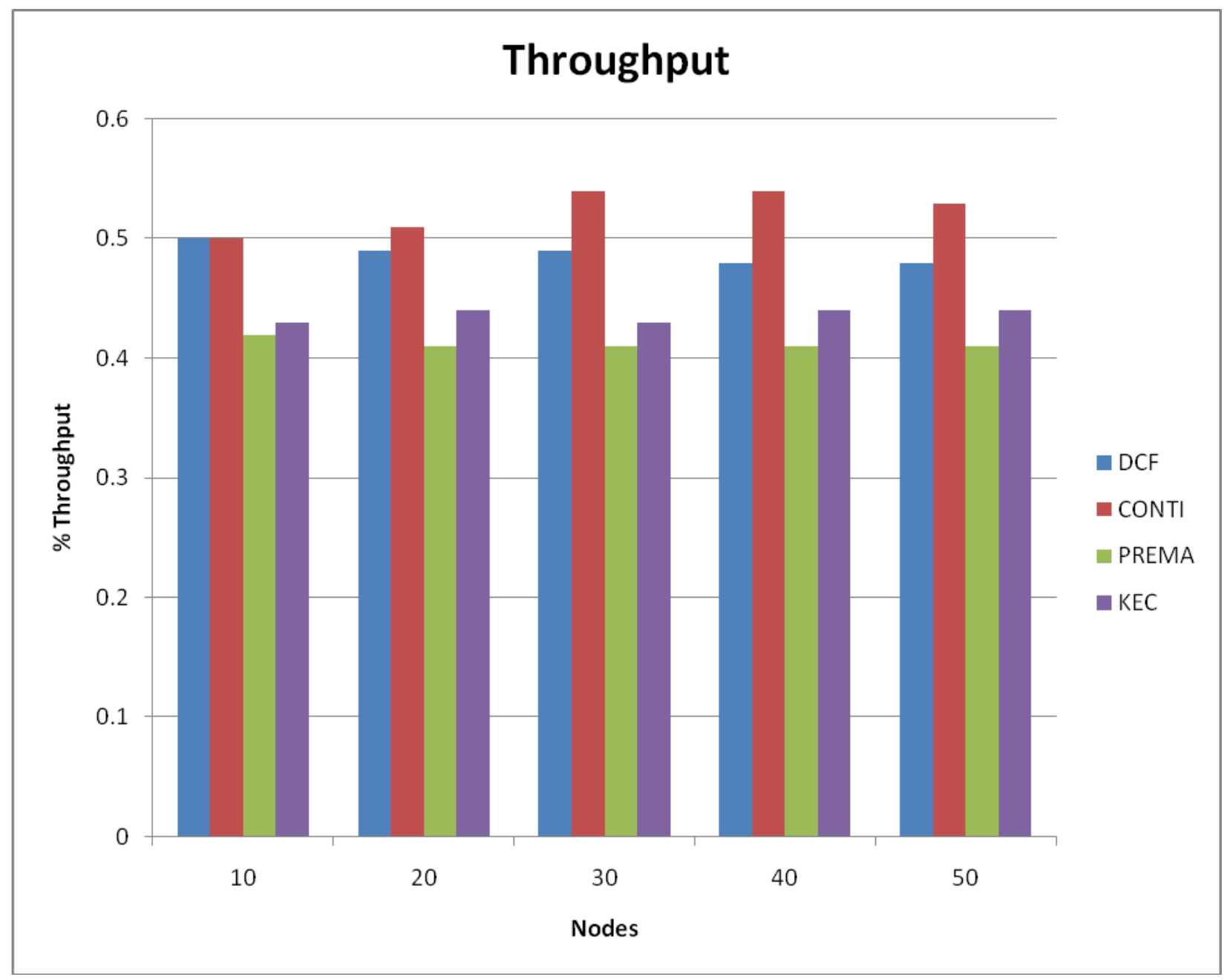

Fig 4.3: Bar chart for comparison of throughput for 10, 20, 30, 40 and 50 nodes for DCF, CONTI, PREMA and k-EC

From these plots we can draw a few conclusions. The first observation is that CONTI has the best performance in terms of throughput. DCF has similar performance as CONTI for low number of nodes but, its performance starts to fall as the number of nodes increases due to high collision rates. Secondly, PREMA and k-EC are behind DCF and CONTI in terms of throughput. However, k-EC's throughput is slightly higher than that of PREMA as k-EC requires less slots with more number of stations.

\subsection{Average time wasted in contention resolution}


Average time wasted in contention is the amount of time spent or wasted in resolving the contention for each successful packet transmission. The lesser the time wasted in resolving the contention, the better is the contention resolution scheme.

The plot that follows shows our simulation results for the average time wasted in contention for DCF, CONTI, PREMA and k-EC.

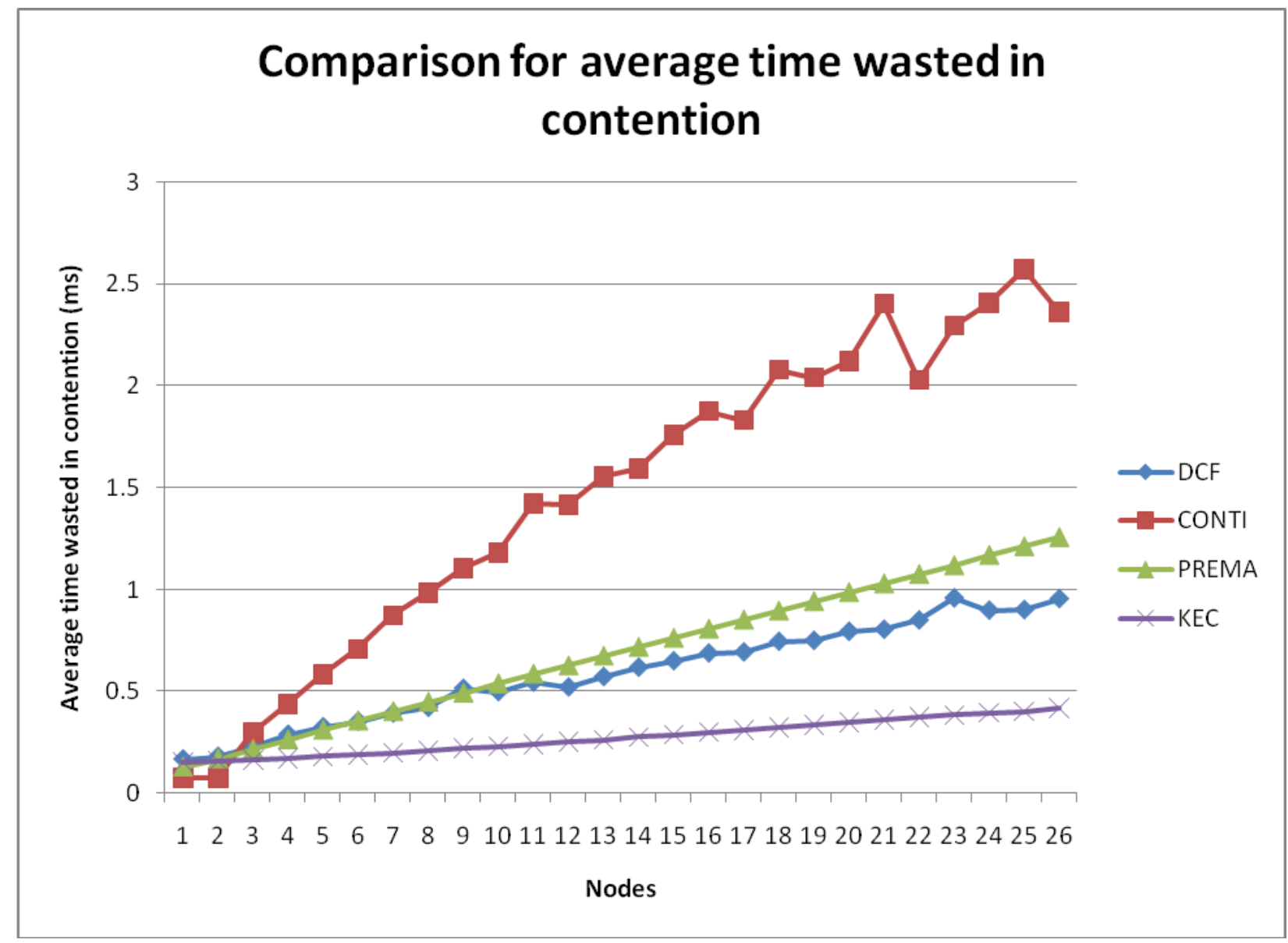

Fig 4.4: Plot for average time wasted in contention for DCF, CONTI, PREMA and k-EC from 1 to 50 nodes 


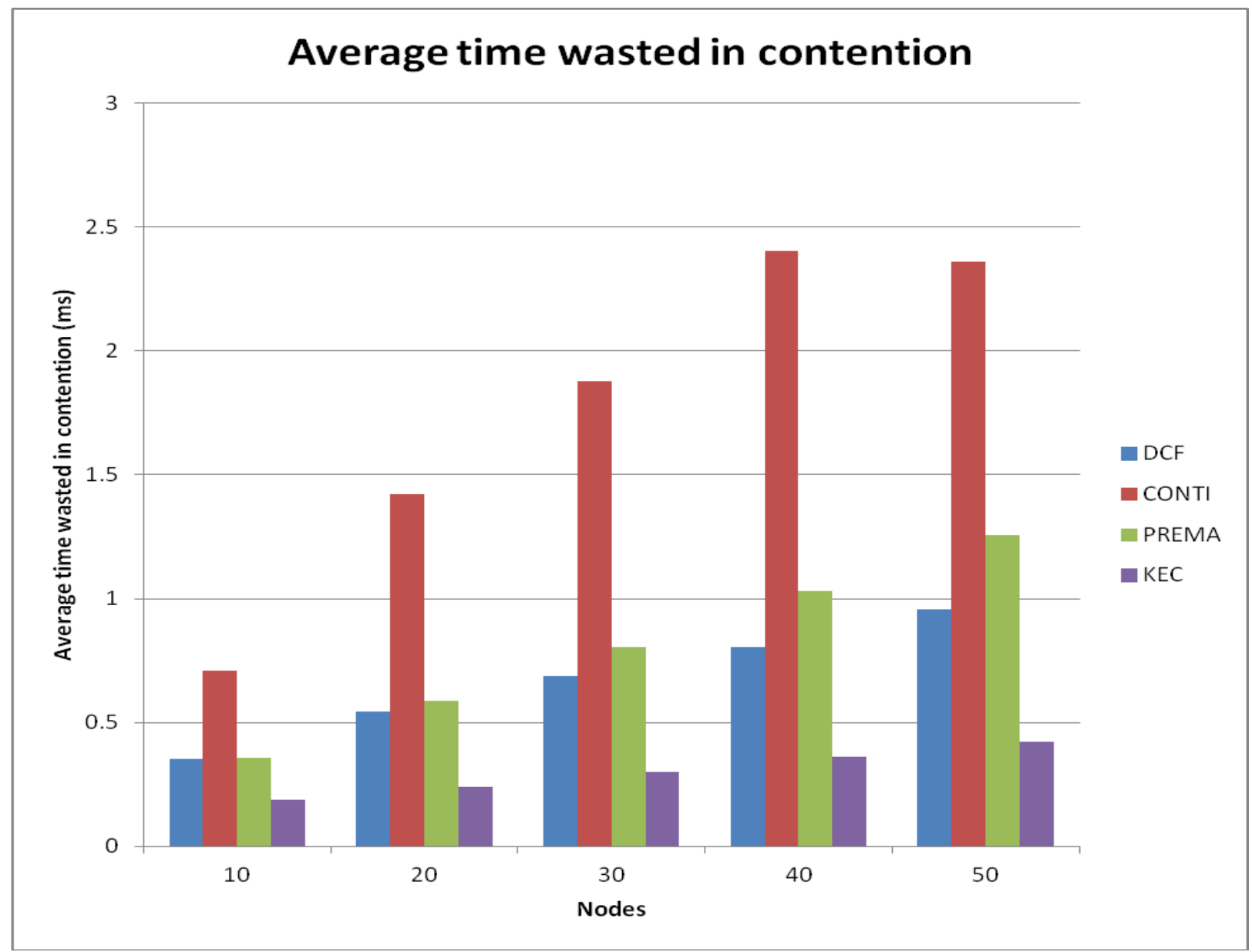

Fig 4.5: Bar chart for comparison of average time wasted in contention for 10, 20, 30, 40 and 50 nodes for DCF, CONTI, PREMA and k-EC

From the plots we can see that CONTI wastes the highest amount of time in contention resolution and this value rises steeply with increasing number of nodes. DCF and PREMA have somewhat similar results although at the very beginning with only a few numbers of nodes all the four contention resolution schemes have similar values for time wasted in contention. k-EC shows the best performance as it wastes the lowest amount of time in contention resolution. 


\subsection{Delay}

We also evaluated the delay of the four contention resolution schemes. The following figures present a comparison of the average delay.

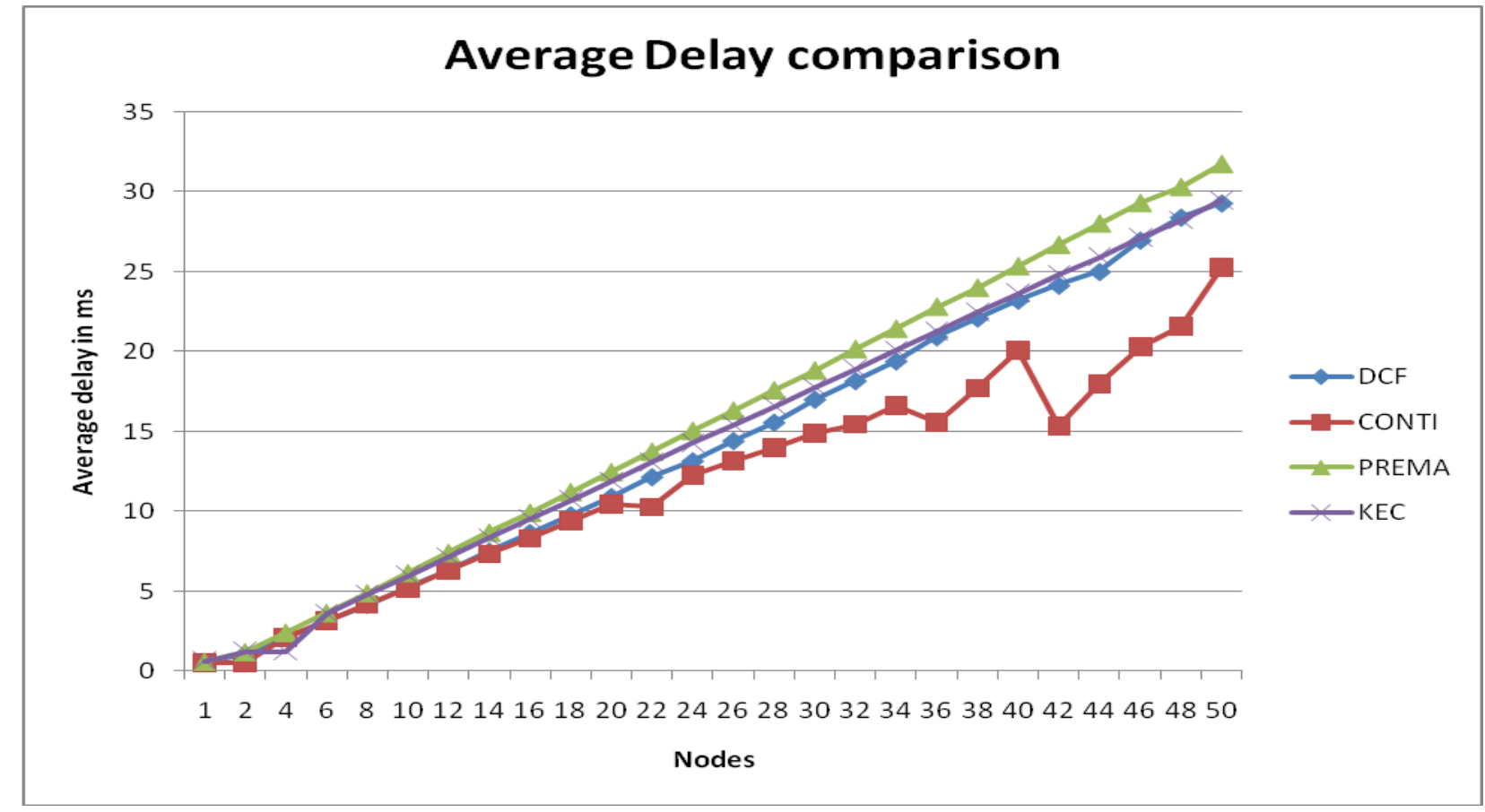

Fig 4.6: Plot for average delay for DCF, CONTI, PREMA and k-EC from 1 to 50 nodes

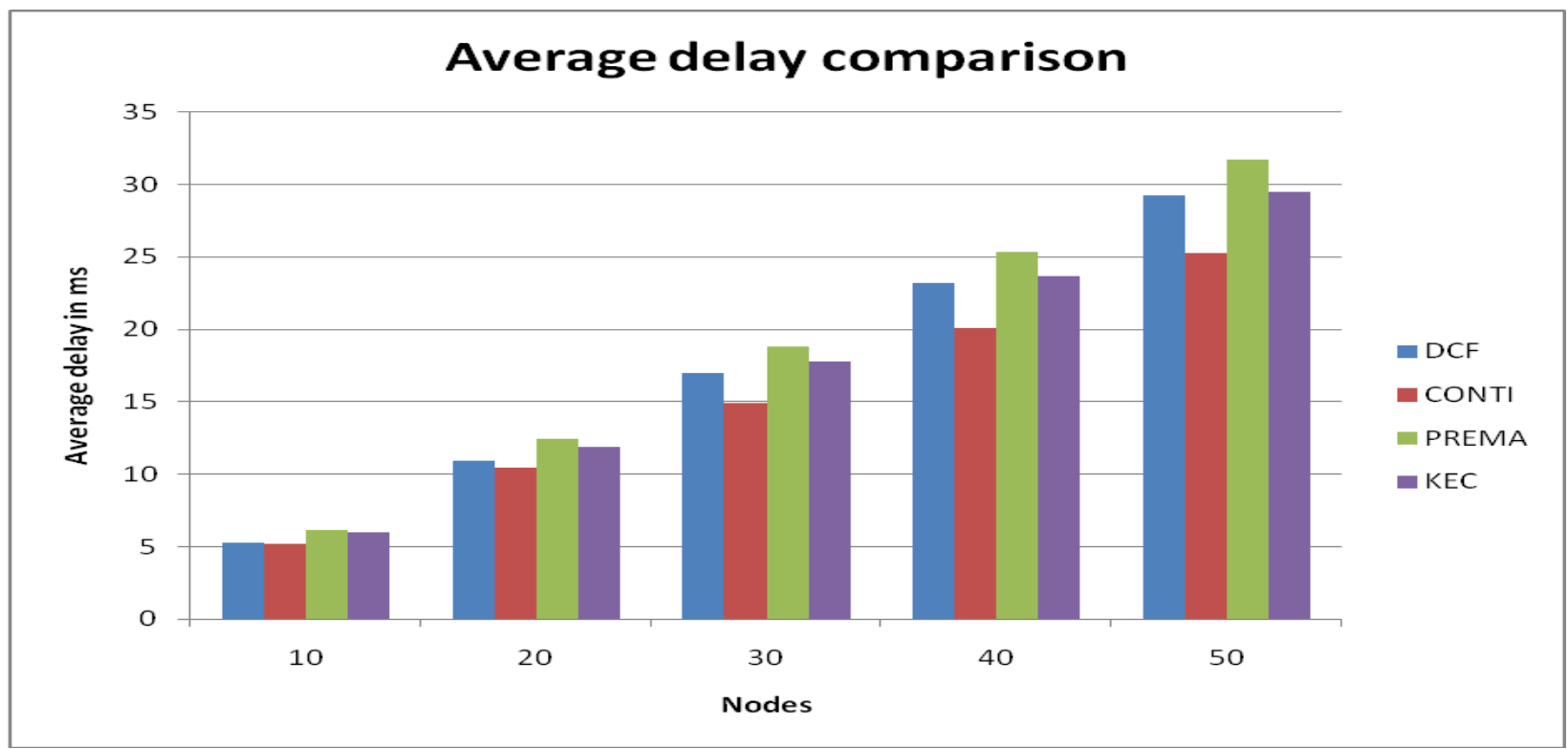

Fig 4.7: Bar chart for comparison of average delay for 10, 20, 30, 40 and 50 nodes for DCF, CONTI, PREMA and k-EC 
The figures show that the delay is increasing as the number of contending nodes increases, as expected. This is because as the number of contending nodes increases the frequency of packet transmission decreases. With as small as 10 nodes, DCF and CONTI have the smallest delay because they have low collision rates and small number of slots. With increasing number of nodes the average delays of DCF, PREMA and k-EC starts increasing. CONTI has a small advantage in the delay values over the other schemes.

\subsection{Summary of observations}

From all the above plots and the conclusions that we have drawn, we can summarize that CONTI is the best contention resolution scheme compared to DCF, PREMA and k-EC. In terms of throughput and Delay, CONTI has shown a very good performance as it had the highest throughput and lowest delay. Although k-EC overrules CONTI in terms of the collision rate and average time spent in contention, the main aim of a contention resolution scheme is to decrease collision but keep the throughput as high as possible which, CONTI has managed to achieve. What CONTI loses in average time spent in contention it more than makes up for it in terms of collision rate, throughput and average delay.

\section{The Unsaturated Model}

The results preceding until this section was for the saturated model of a network where all the nodes in the network have a data packet that it wants to transmit. In other words, the queue of a node is assumed to be full at all times. In this part of the paper we propose an unsaturated model which is close to a real networking scenario where a given node does not always have a packet to send. The queue of a node will have a packet as soon as one is generated. In this case, we modify our codes so that a node will first check its queue for a packet that needs to be transmitted before sensing the medium idle for DIFS.

We have modelled this scenario by making use of a data structure called the arraylist which simulates the queue of a node. The generation of a packet follows a negative exponential distribution with parameter called a 'mean offtime'. An offtime using this mean is chosen by the data generator of each node and it waits for this amount of time before pushing a data packet into the node's queue. The data generator and node entities in the simulation environment work simultaneously and independently to to be able to work according to our proposed model. 
We study the behaviour of the contention resolution schemes in three different scenarios where the mean offtimes are 100, 1000 and $10000 \mu$ s respectively. We draw conclusions from the simulations as follows.

\subsection{Simulation Results}

In this section, we present the results of our simulation in the unsaturated model for DCF, CONTI, PREMA and k-EC. We draw a comparison between the percentage collision, throughput, delay, average time wasted in contention and average queueing delay for three different mean offtimes.

\subsection{Mean Offtime $=100 \mu \mathrm{s}$}

(a) Collision rate

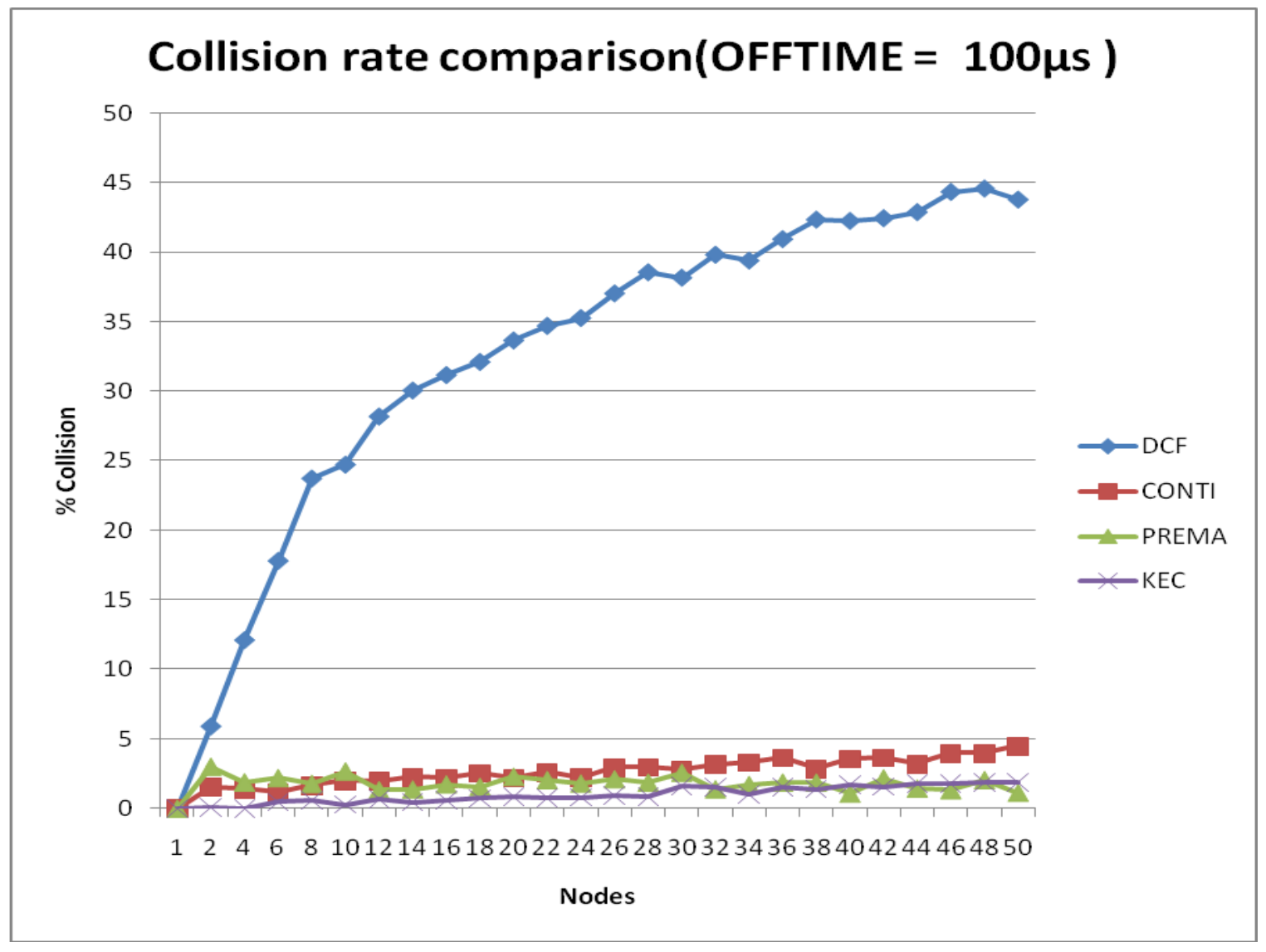

Fig 5.1: Plot for collision rate with mean offtime $=100 \mu$ s for DCF, CONTI, PREMA and k-EC from 1 to 50 nodes

As expected, the collision rate with mean offtime $=100 \mu_{\mathrm{s}}$ is similar to the saturated model as the generation time of the packets is very small. The plot shows that DCF has the highest rate of collision. 
(b) Throughput

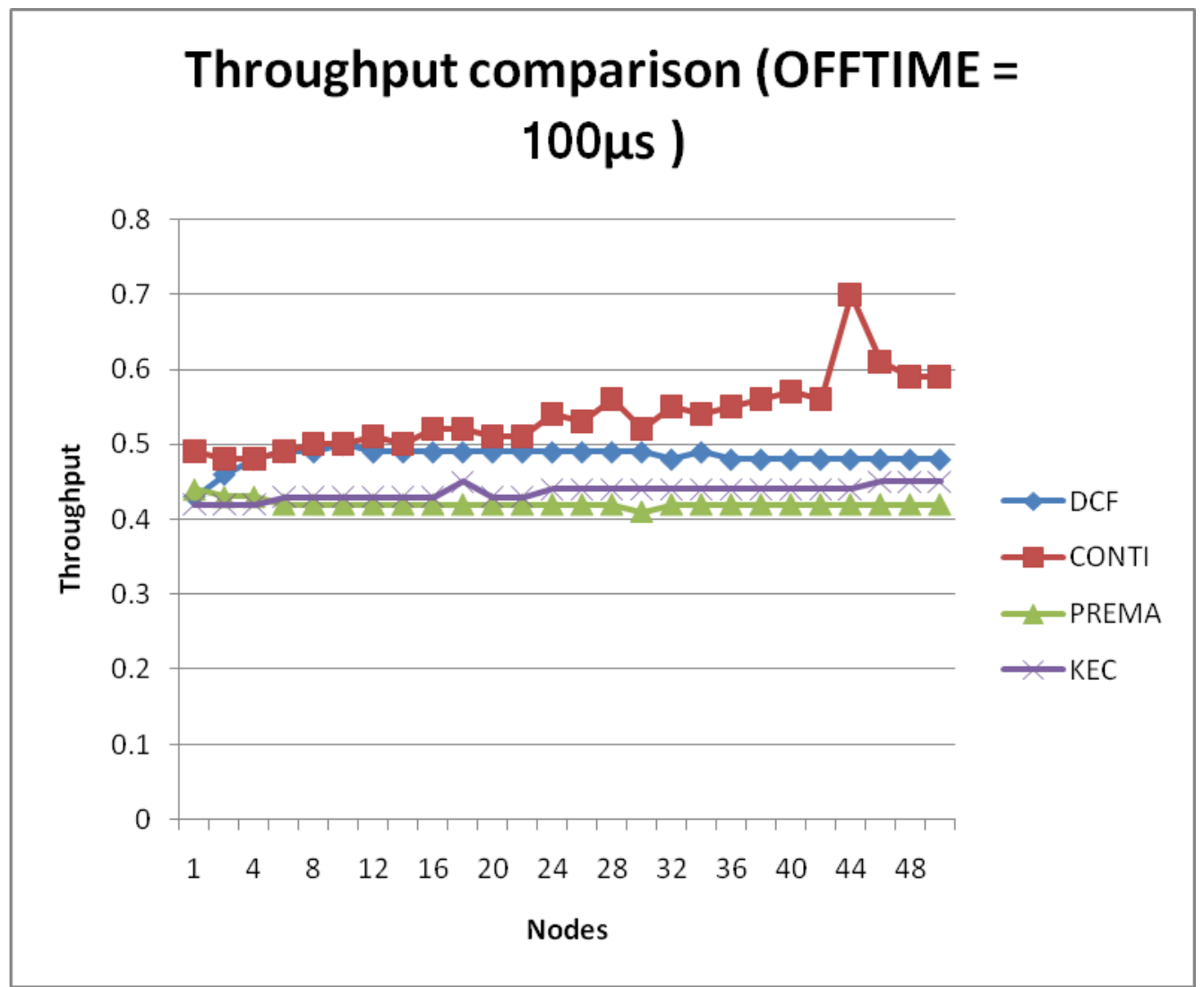

Fig 5.2: Plot for throughput with mean offtime $=100 \mu$ s for DCF, CONTI, PREMA and kEC from 1 to 50 nodes 


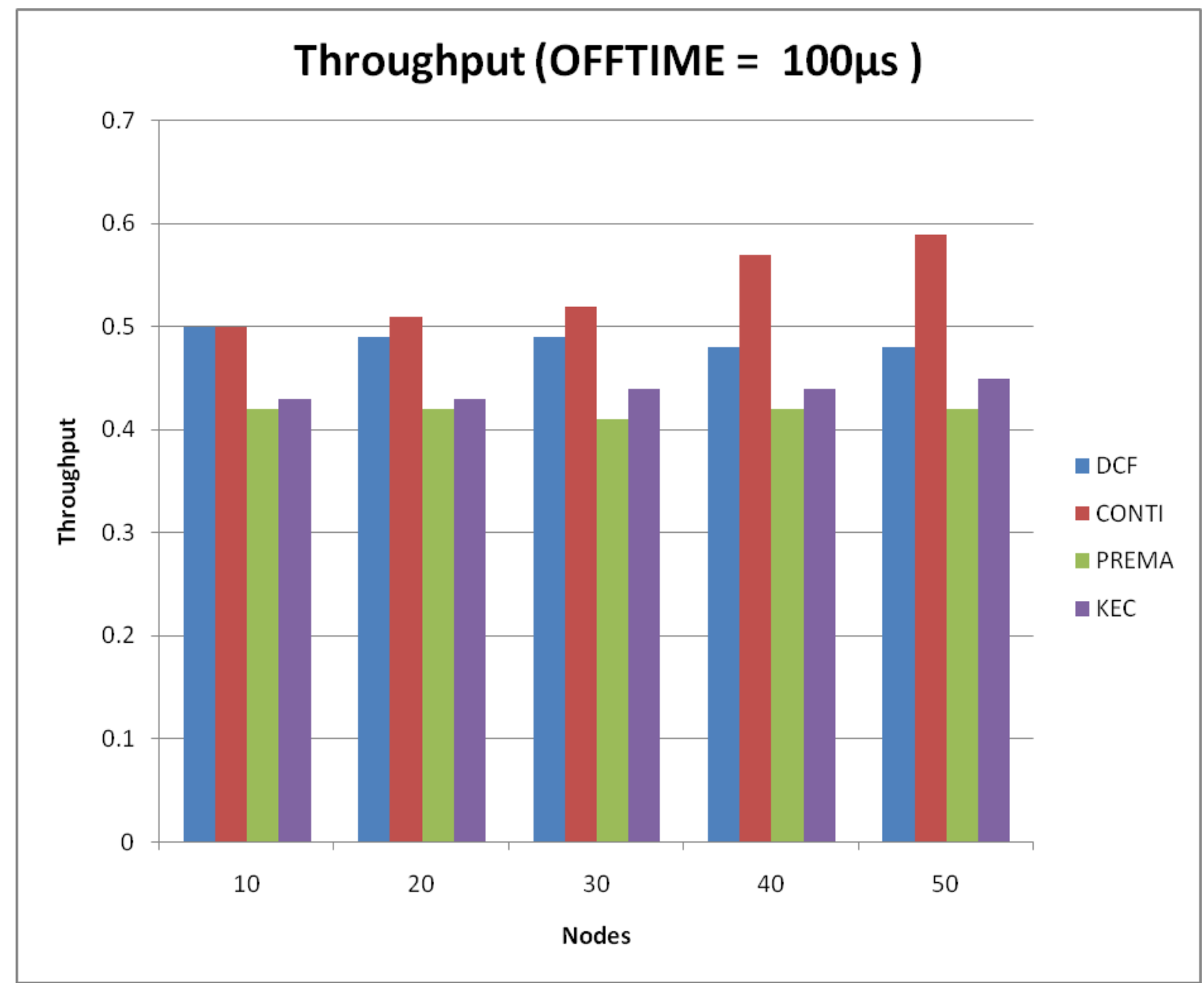

Fig 5.3: Bar chart for comparison of throughput for 10, 20, 30, 40 and 50 nodes for DCF, CONTI, PREMA and k-EC with mean offtime $=100 \mu \mathrm{s}$

With the number of nodes as small as 10, the throughput of DCF and CONTI are similar. As the number of nodes increases the performance of DCF degrades as the collision rate increases. CONTI has the highest throughput. PREMA and k-EC has a constant throughput.

(c) Delay 


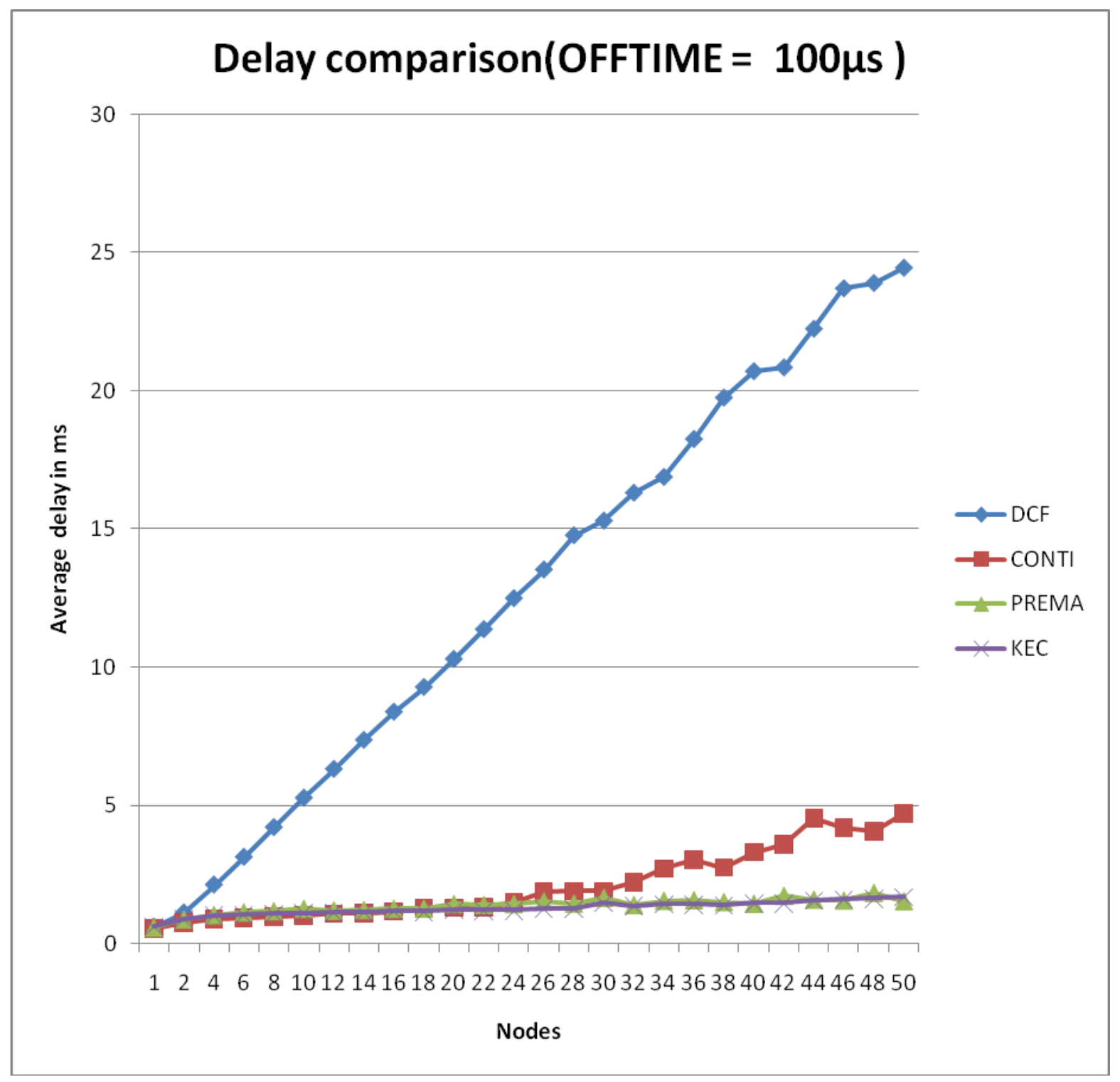

Fig 5.4: Plot for delay comparison with mean offtime $=100 \mu$ s for DCF, CONTI, PREMA and k-EC from 1 to 50 nodes 


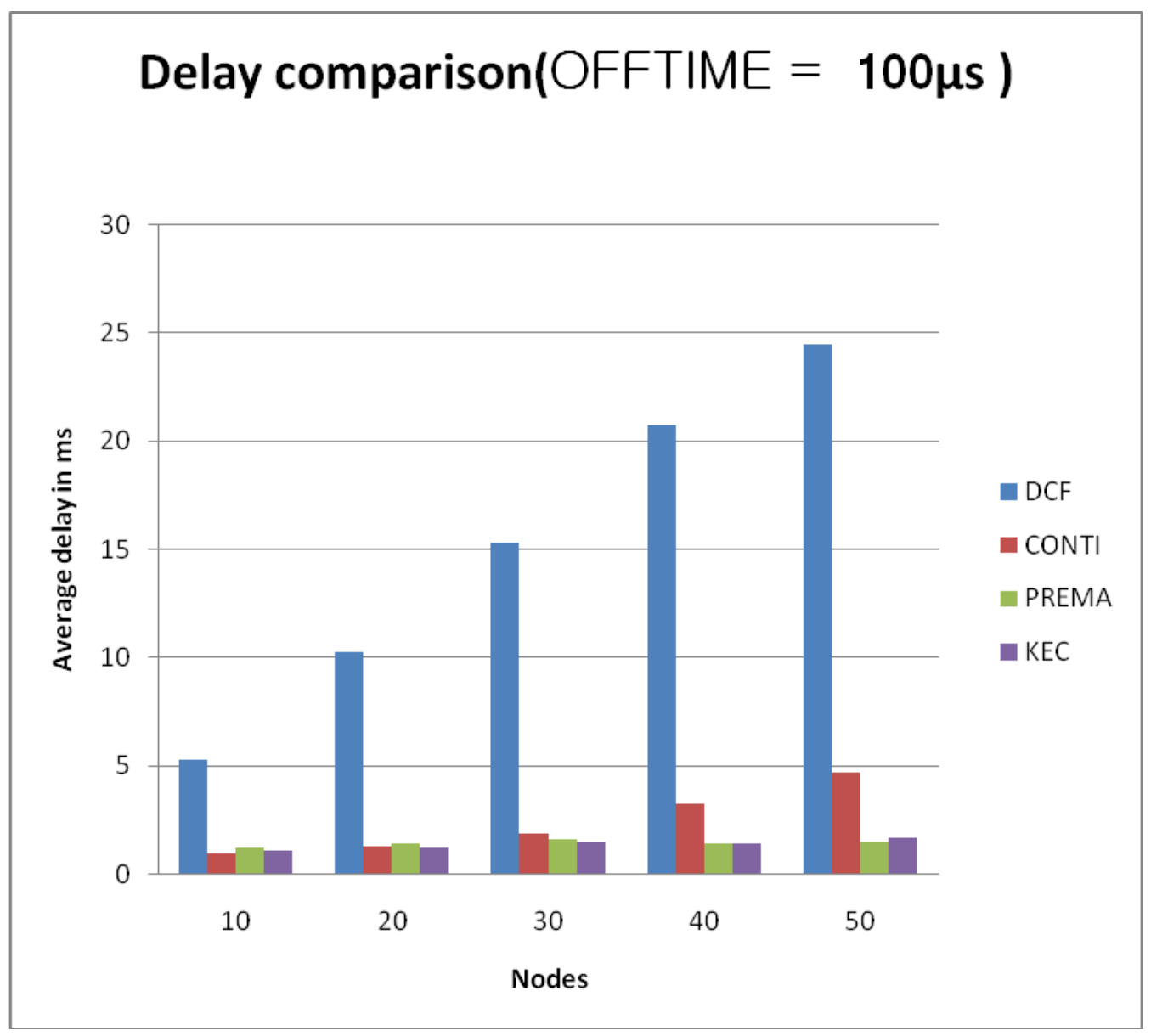

Fig 5.5: Bar chart for delay comparison for 10, 20, 30, 40 and 50 nodes for DCF, CONTI, PREMA and k-EC with mean offtime $=100 \mu \mathrm{s}$

Even in the unsaturated model, DCF has the highest amount of delay with the exception that PREMA and k-EC now have a lower delay than CONTI.

\section{(d) Average time wasted in contention}

For the unsaturated model, we also calculated the average time wasted in contention and the lower this time, the better the resolution scheme. 


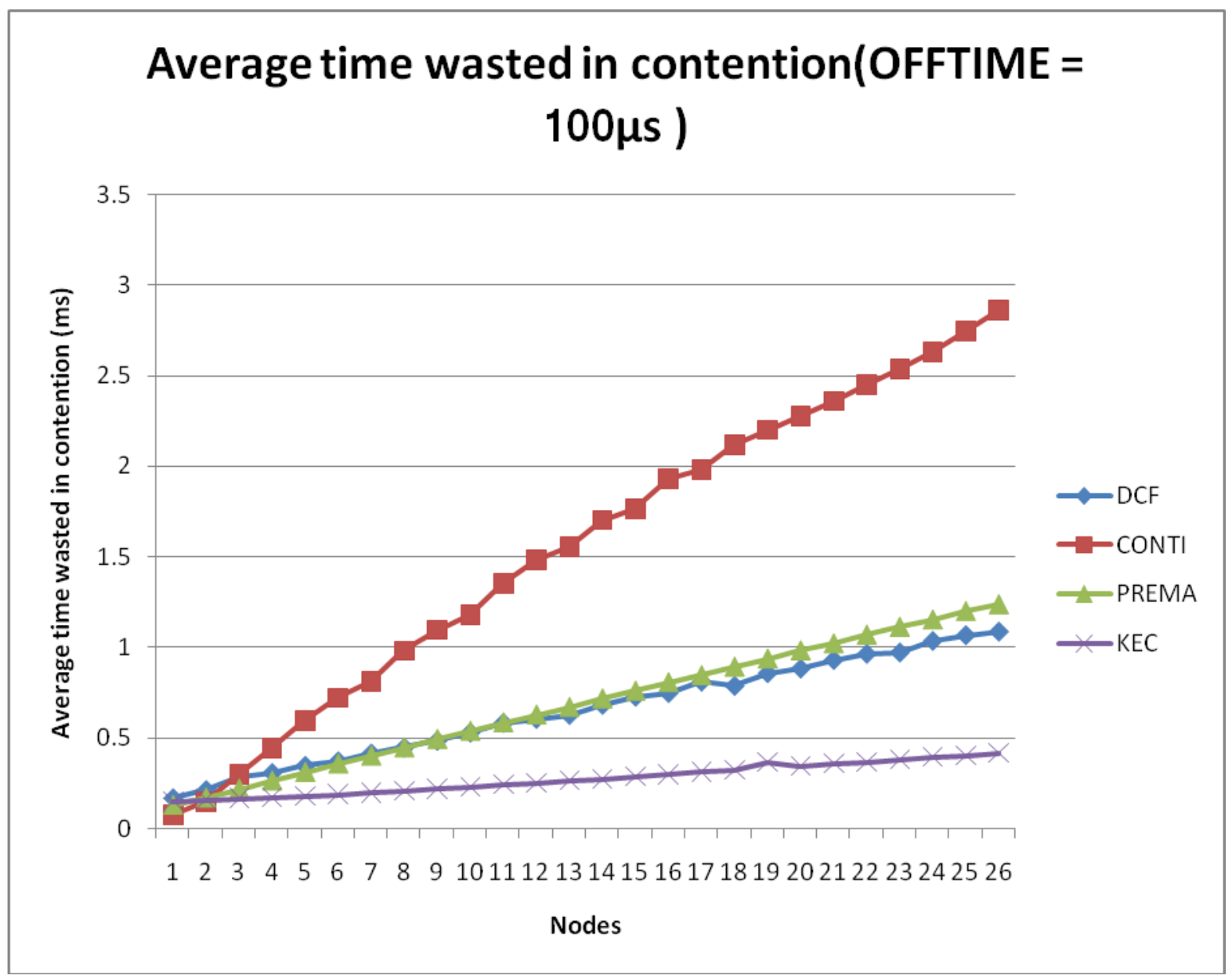

Fig 5.6: Plot for average time wasted in contention with mean offtime $=100 \mu$ s for DCF, CONTI, PREMA and k-EC from 1 to 50 nodes 


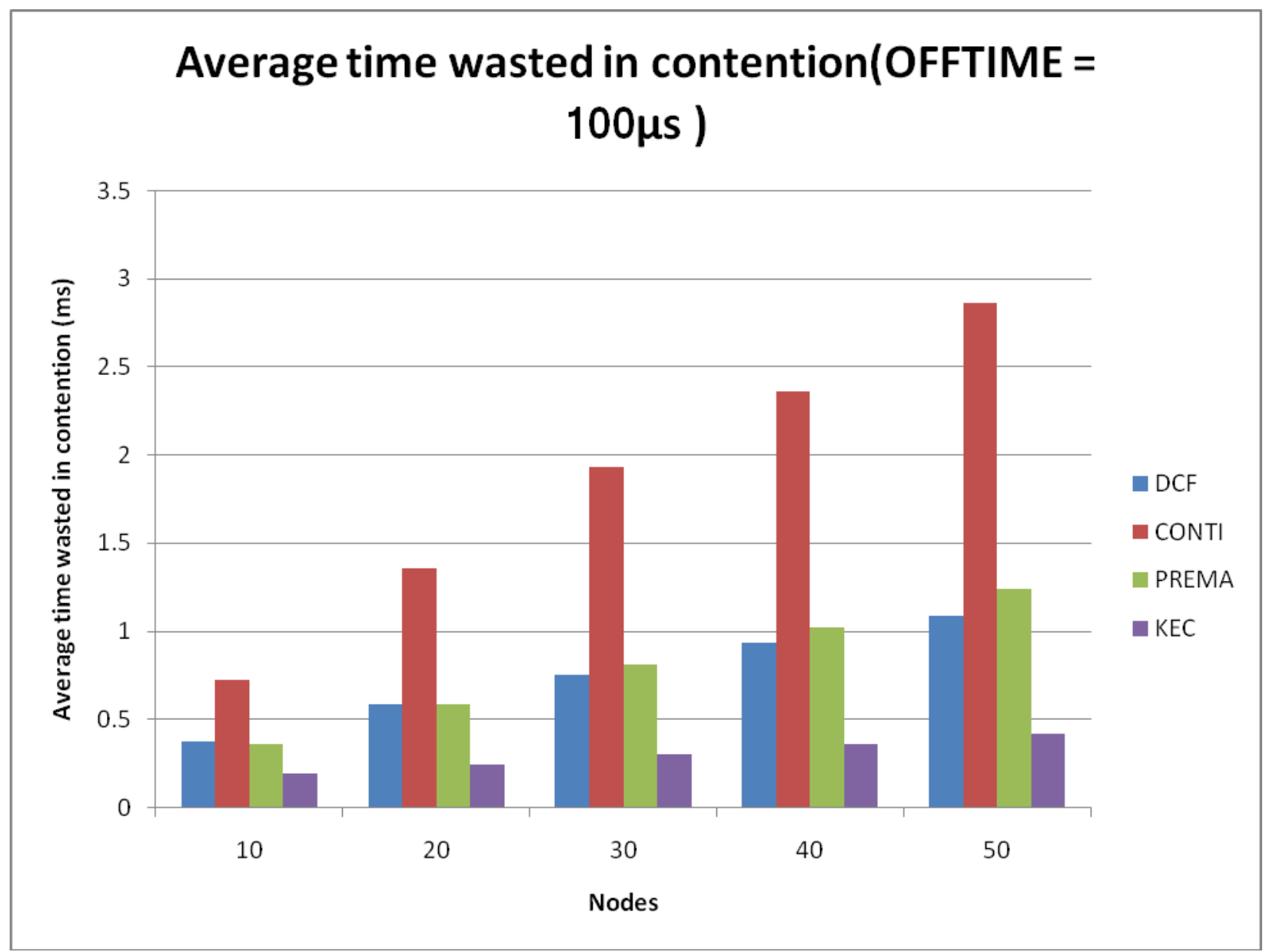

Fig 5.7: Bar chart for average time wasted in contention for 10, 20, 30, 40 and 50 nodes for DCF, CONTI, PREMA and k-EC with mean offtime $=100 \mu \mathrm{s}$

CONTI wastes the highest amount of time in contention as it sends jam for the entire seven slots. On the other hand, DCF and PREMA have similar results. k-EC wastes the least amount of time in contention.

\section{(e) Average queueing delay}

Queuing delay is the time a packet spends in the queue. This time is measured from when the packet is pushed into the queue up until it is successfully transmitted. The lesser the queuing delay, the better the contention scheme. 


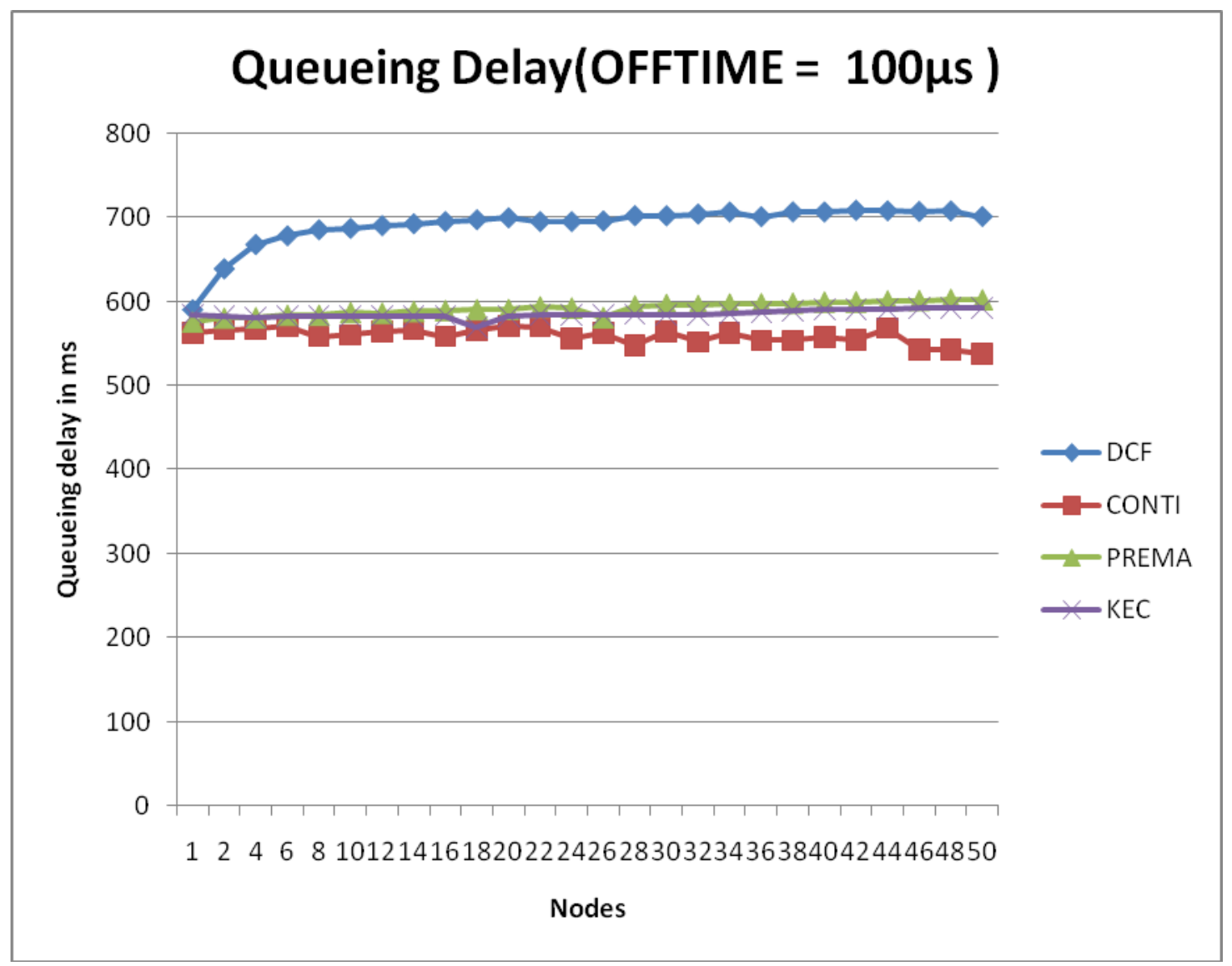

Fig 5.8: Plot for queuing delay with mean offtime $=100 \mu$ s for DCF, CONTI, PREMA and k-EC from 1 to 50 nodes 


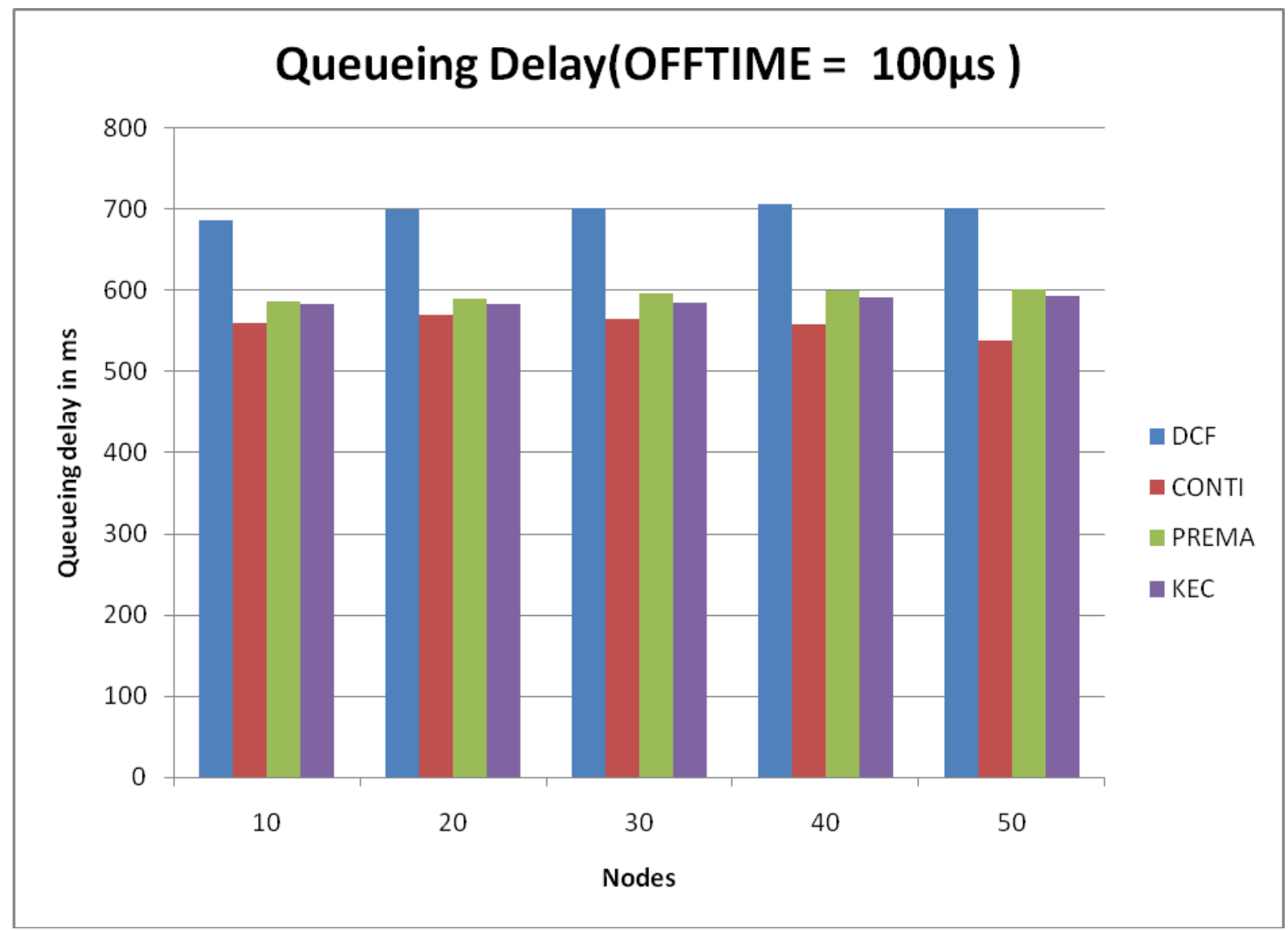

Fig 5.9: Bar chart for queuing delay for 10, 20, 30, 40 and 50 nodes for DCF, CONTI, PREMA and $\mathrm{k}-\mathrm{EC}$ with mean offtime $=100 \mu \mathrm{s}$

From the plots we can see that DCF has the worst performance as the packets have to wait for a long time in queue. K-EC and PREMA show similar performance. CONTI has the best performance. 


\subsection{Mean Offtime $=1000 \mu \mathrm{s}$}

\section{(a) Collision rate}

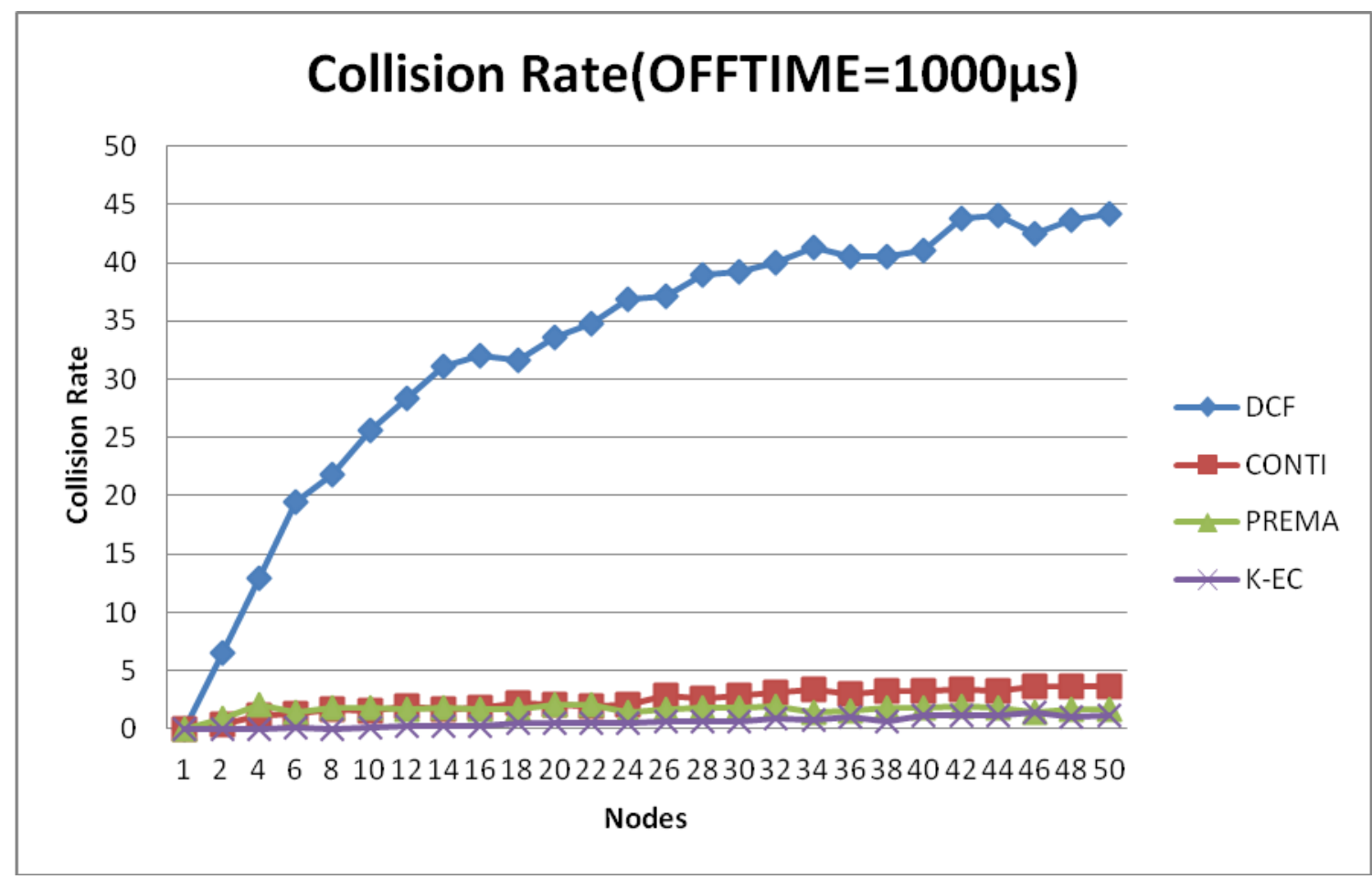

Fig 5.10: Plot for collision rate with mean offtime $=1000 \mu$ s for DCF, CONTI, PREMA and k$\mathrm{EC}$ from 1 to 50 nodes

For mean offtime $=1000 \mu \mathrm{s}$, we can see that DCF has the highest rate of collision. CONTI, PREMA and $\mathrm{k}-\mathrm{EC}$ show almost the same collision rate.

(b) Throughput 


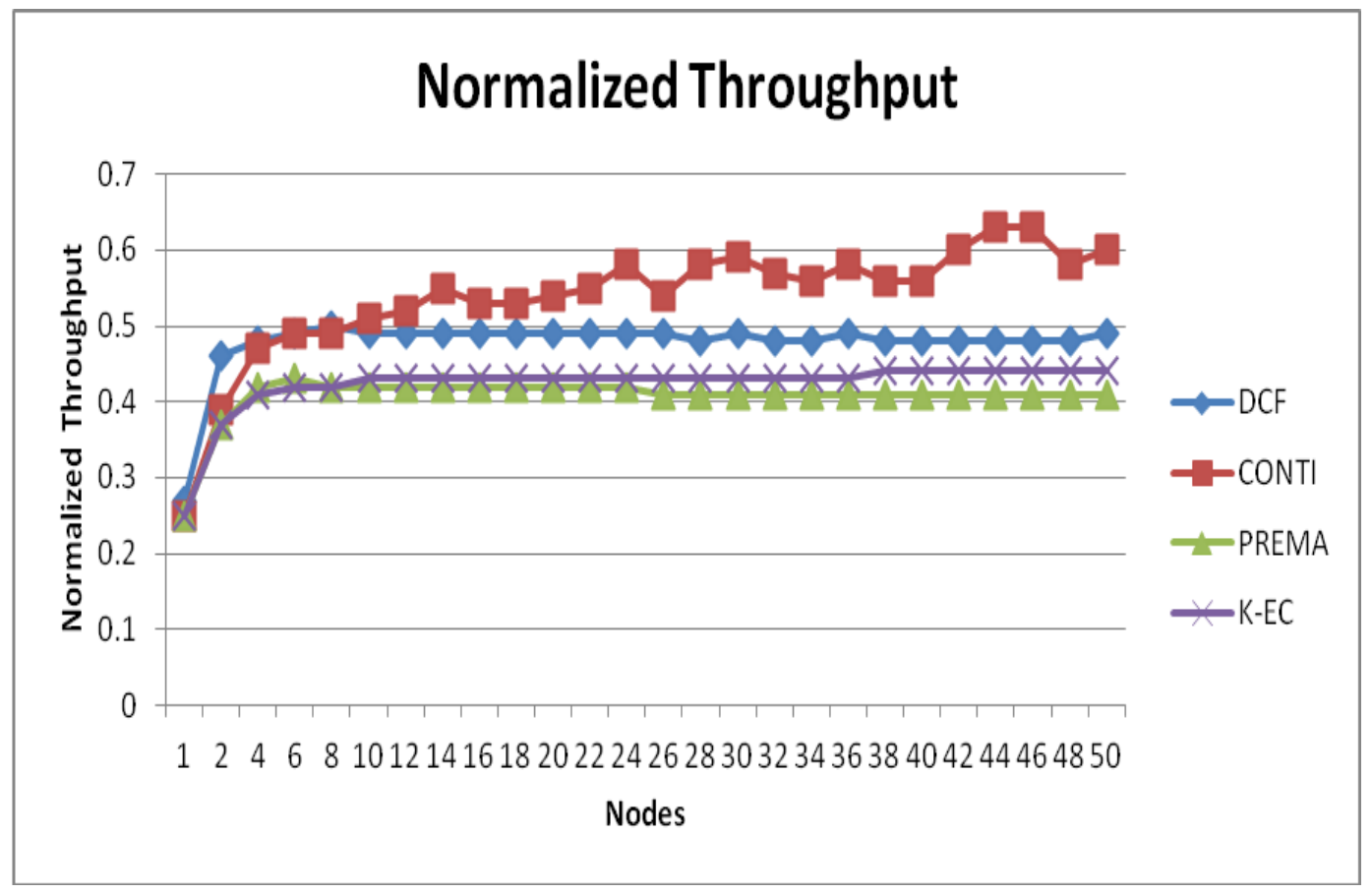

Fig 5.11: Plot for throughput with mean offtime $=1000 \mu$ s for DCF, CONTI, PREMA and k-EC from 1 to 50 nodes

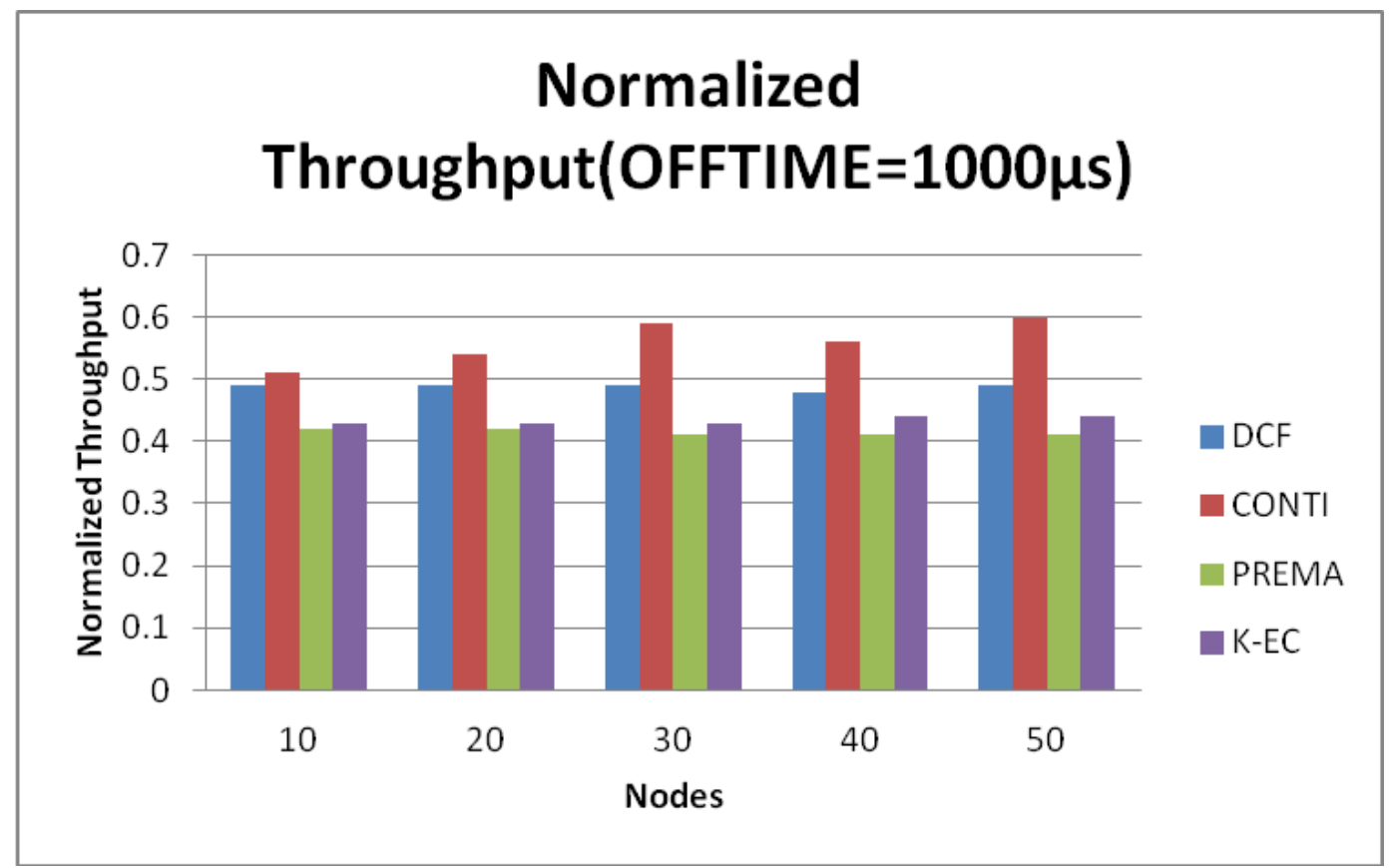

Fig 5.12: Bar chart for comparison of throughput for 10, 20, 30, 40 and 50 nodes for DCF, CONTI, PREMA and k-EC with mean offtime $=1000 \mu \mathrm{s}$ 
When the mean offtime $=1000 \mu \mathrm{s}$, the above plots show the throughput comparison of DCF, CONTI, PREMA and k-EC. When the number of nodes is small DCF and CONTI have similar throughput values. As the number of nodes increases, the throughput of DCF decreases as the collision rate increases. PREMA and k-EC have similar throughput values throughout. However, $\mathrm{k}$-EC has an advantage over PREMA as it can eliminate maximum number of nodes in one elimination round. Overall, CONTI has the highest throughput.

(c) Delay

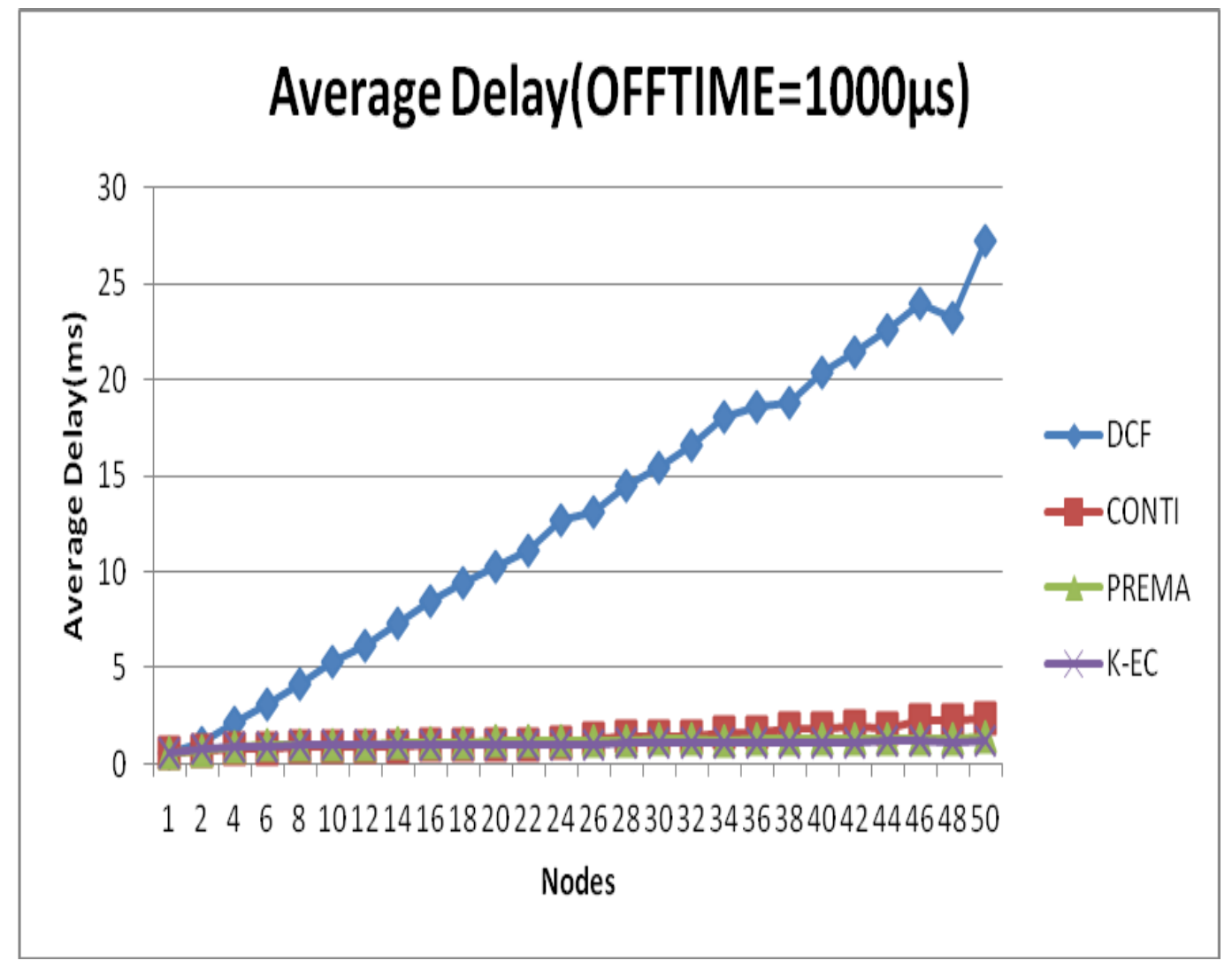

Fig 5.13: Plot for average delay with mean offtime $=1000 \mu$ s for DCF, CONTI, PREMA and kEC from 1 to 50 nodes 


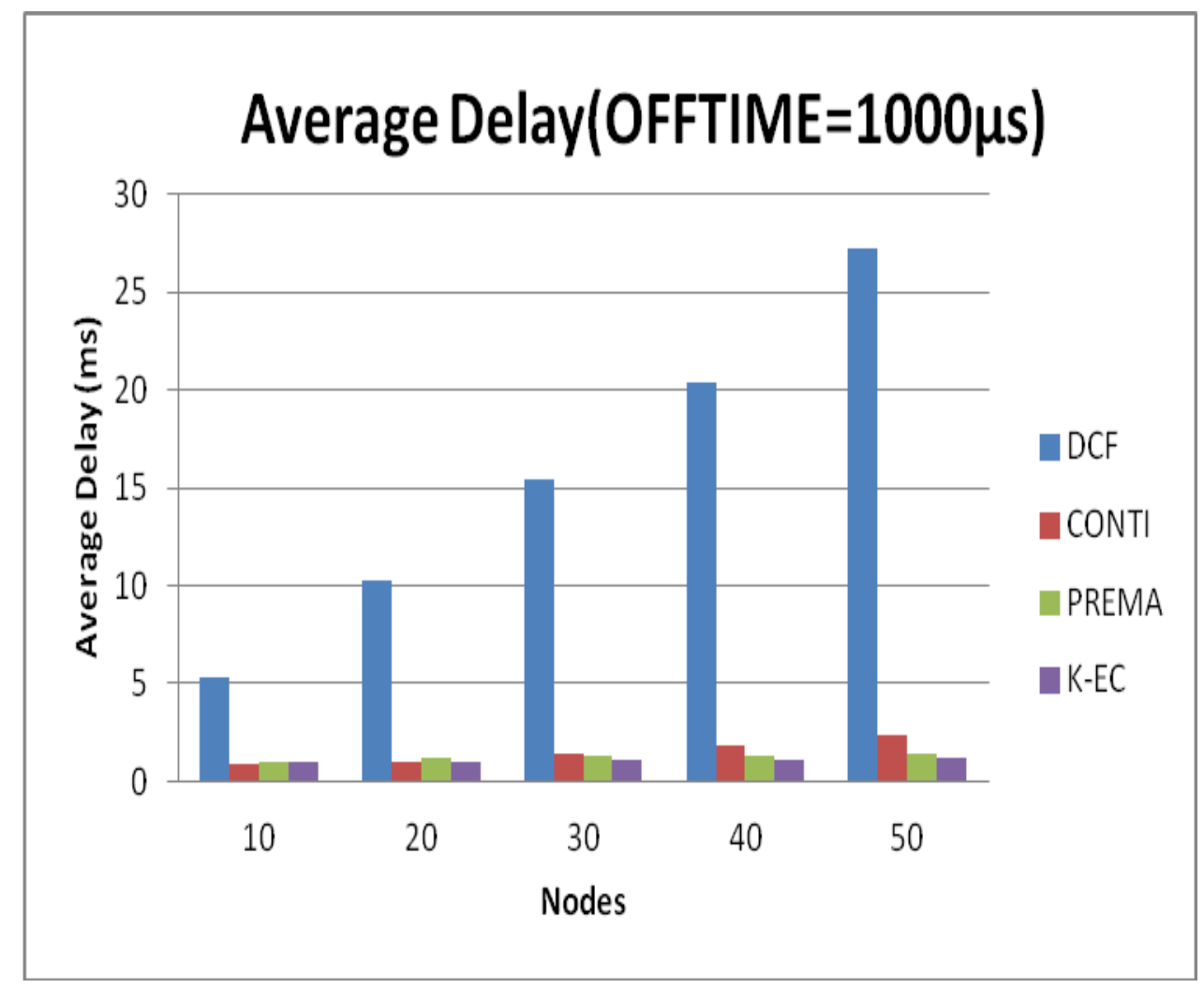

Fig 5.14: Bar chart for comparison of average delay for 10, 20, 30, 40 and 50 nodes for DCF, CONTI, PREMA and k-EC with mean offtime $=1000 \mu \mathrm{s}$

DCF has the highest delay. CONTI, PREMA and k-EC have similar values for delay. With increasing number of nodes, the delay for k-EC decreases.

(d) Average time wasted in contention 


\section{Average Time Wasted in Contention(OFFTIME $=1000 \mu \mathrm{s}$ )}

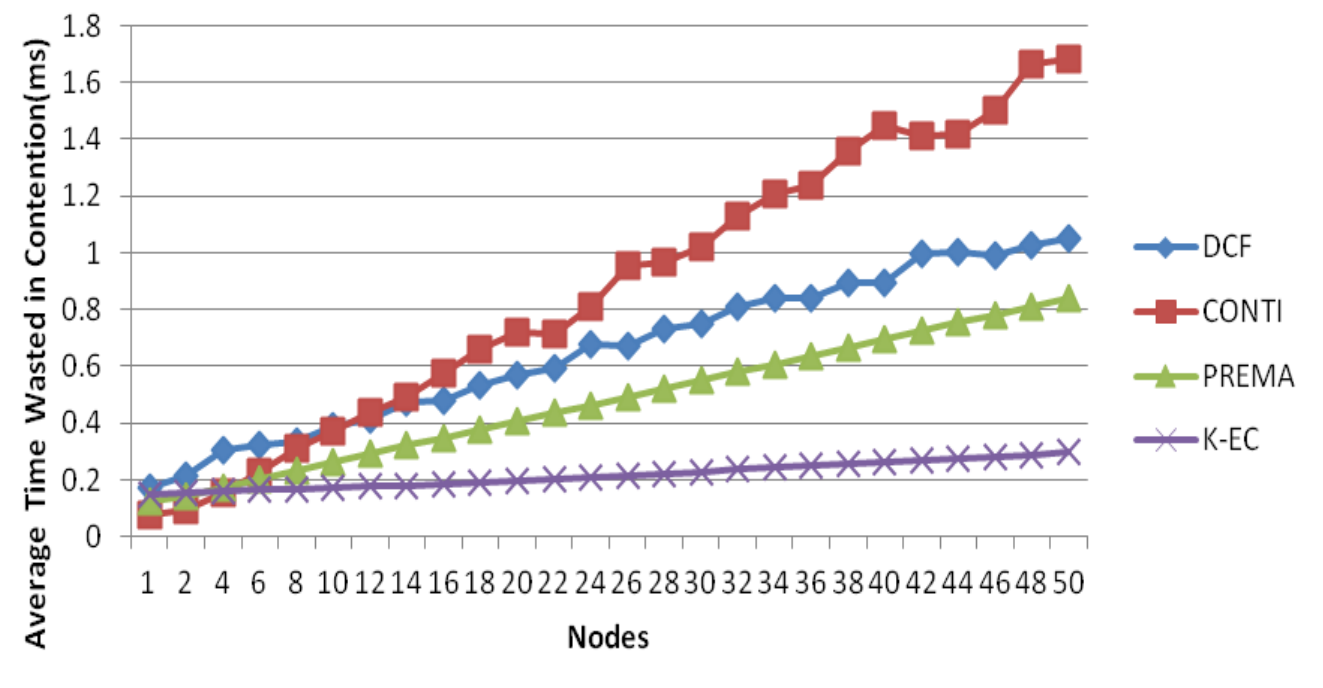

Fig 5.15: Plot for average time wasted in contention with mean offtime $=1000 \mu$ s for DCF, CONTI, PREMA and k-EC from 1 to 50 nodes

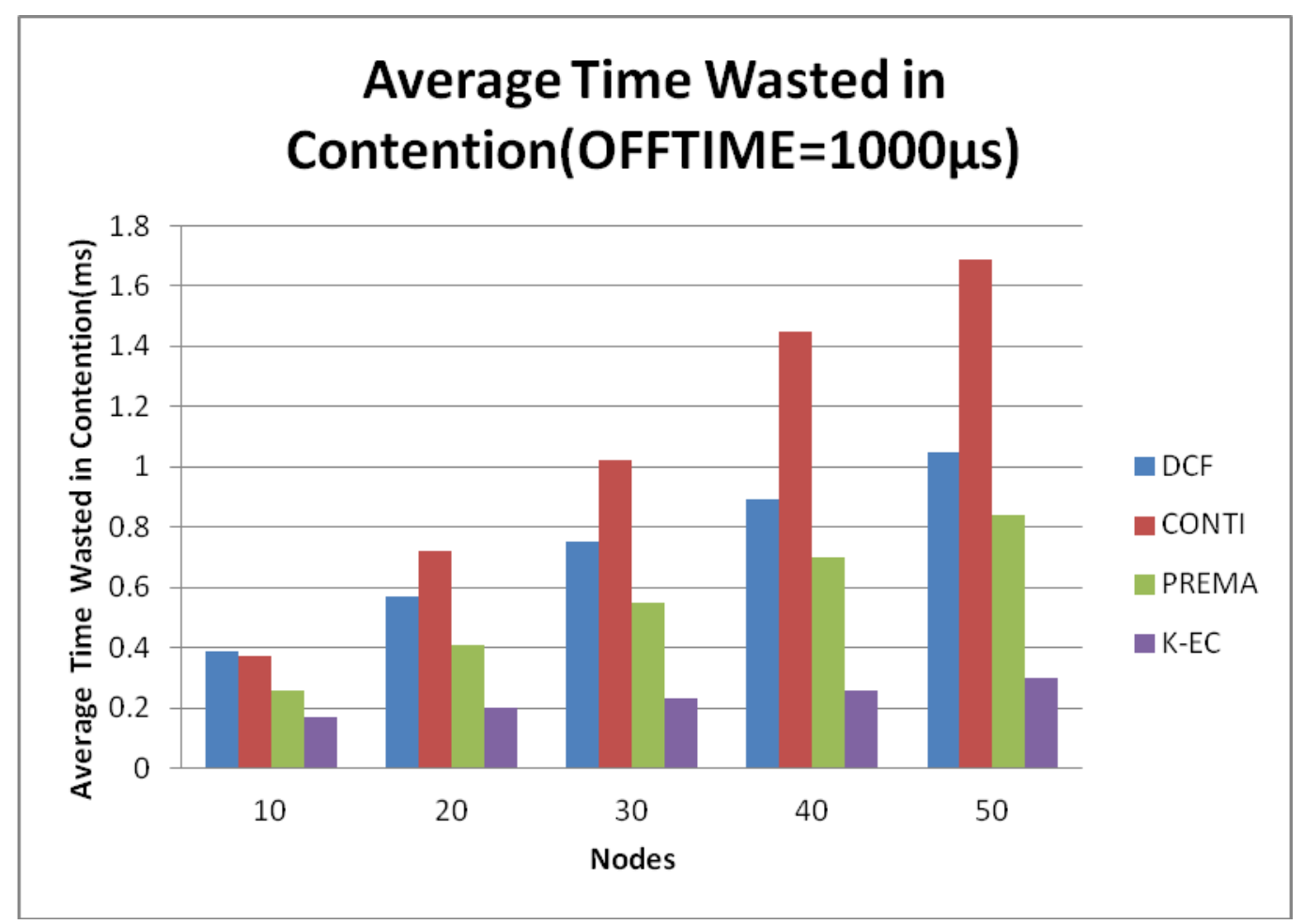

Fig 5.16: Bar chart for average time wasted in contention for 10, 20, 30, 40 and 50 nodes for DCF, CONTI, PREMA and k-EC with mean offtime $=1000 \mu \mathrm{s}$ 
For low number of nodes, all the four schemes waste almost the same amount of time in contention. As the number of nodes increase, CONTI has the highest amount of time wasted in contention.

(e) Average queuing delay

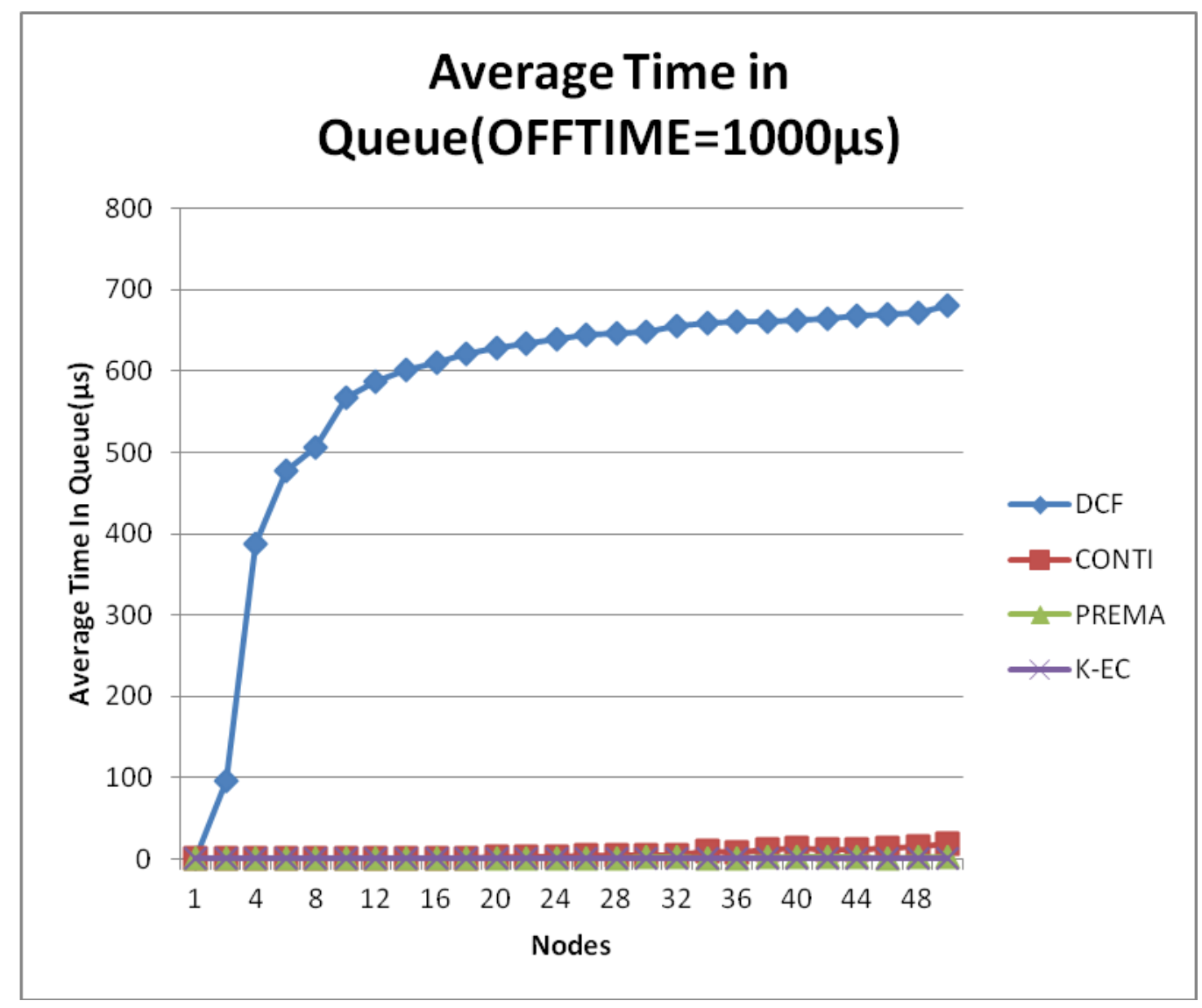

Fig 5.17: Plot for queuing delay with mean offtime $=1000 \mu$ s for DCF, CONTI, PREMA and kEC from 1 to 50 nodes 


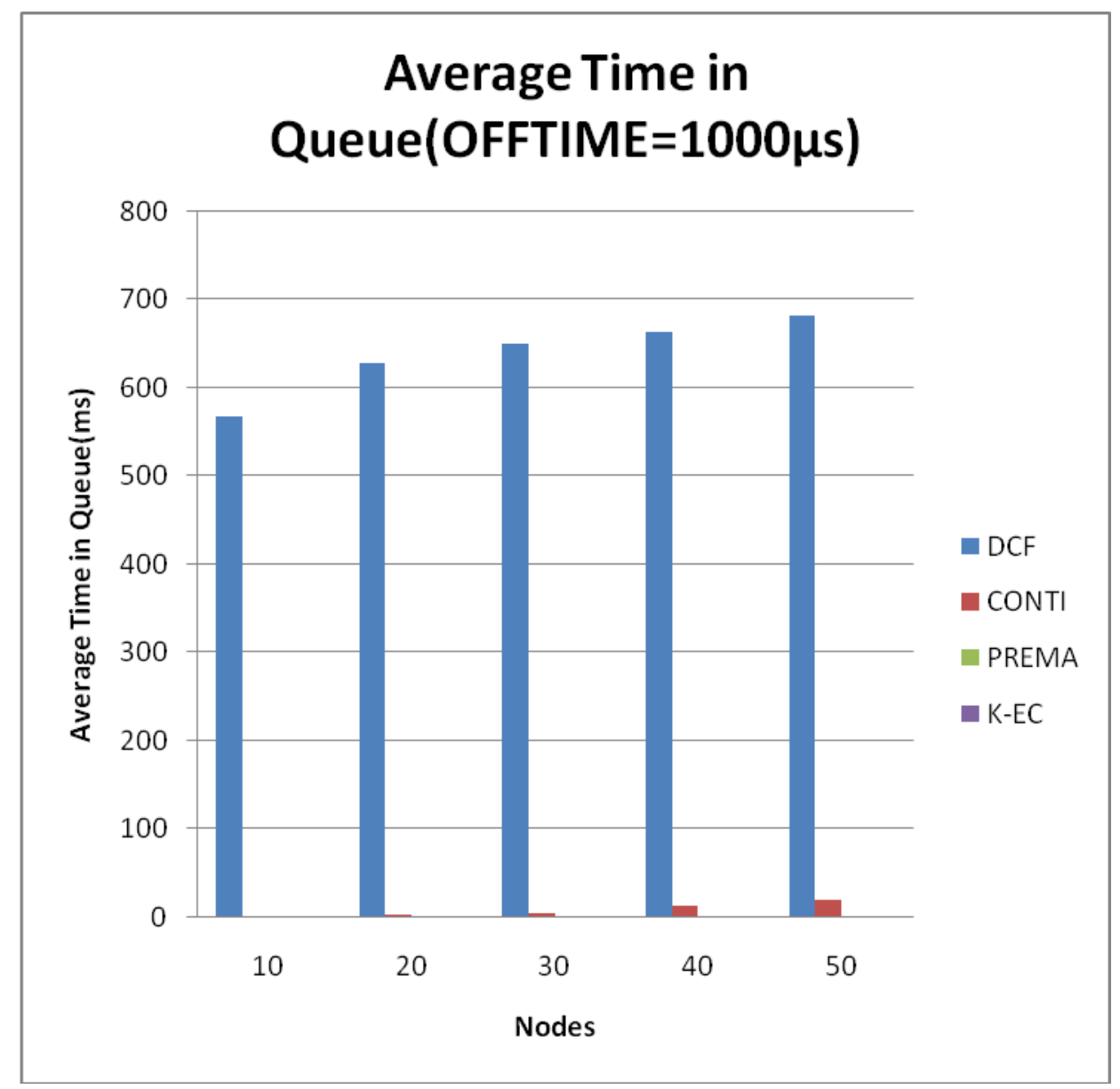

Fig 5.18: Bar chart for queuing delay for 10, 20, 30, 40 and 50 nodes for DCF, CONTI, PREMA and $\mathrm{k}-\mathrm{EC}$ with mean offtime $=1000 \mu \mathrm{s}$

From the plots we can see that DCF has the worst performance as the packets have to spend a very high amount of time in queue before being transmitted. Whereas CONTI, PREMA and k$\mathrm{EC}$, the queuing delay is very low. For DCF, the delay goes up to $680.52 \mu \mathrm{s}$ whereas for k-EC the delay is $1.795 \mu \mathrm{s}$ which is the lowest. 


\subsection{Mean Offtime $=10,000 \mu \mathrm{s}$}

\section{(a) Collision rate}

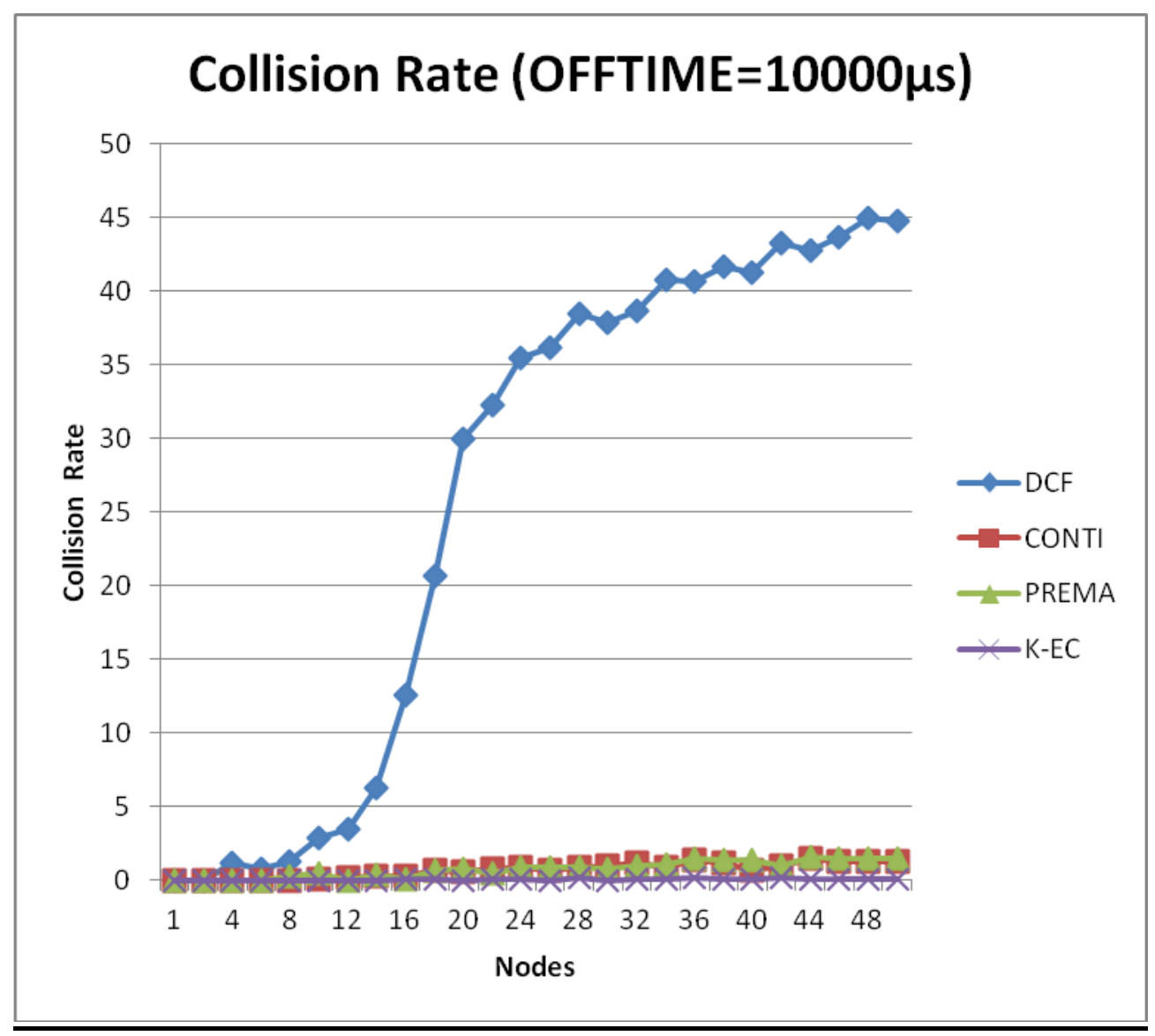

Fig 5.19: Plot for collision rate with mean offtime $=10,000 \mu$ s for DCF, CONTI, PREMA and kEC from 1 to 50 nodes

The lower the collision rate, the better the contention resolution scheme. In the above plots, we can see that DCF has a very high collision rate whereas the collision rates for CONTI, PREMA and k-EC are very low. The collision rate for DCF increases steeply with the increase in number of nodes.

(b) Throughput 


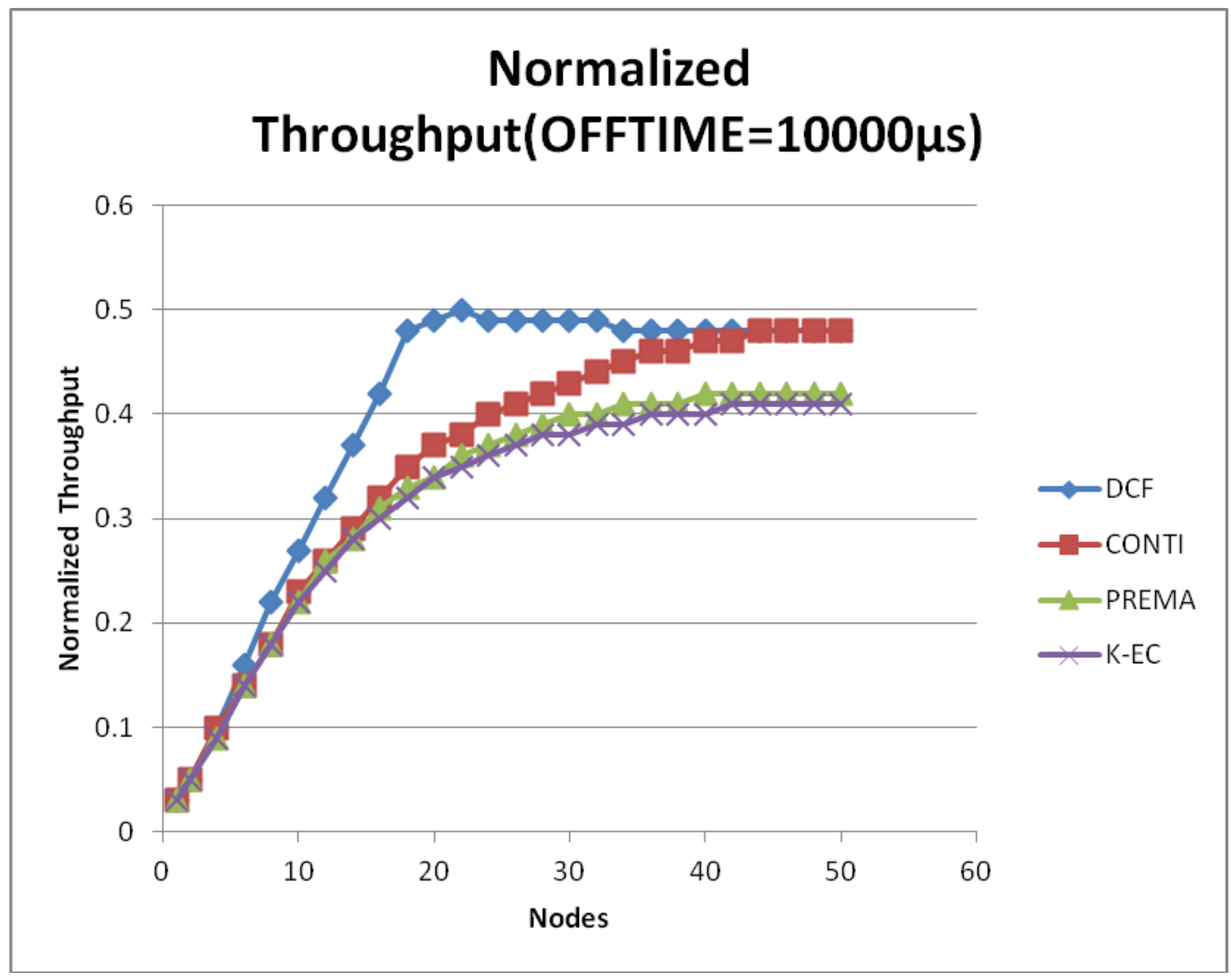

Fig 5.20: Plot for throughput with mean offtime $=10000 \mu$ s for DCF, CONTI, PREMA and k-EC from 1 to 50 nodes 


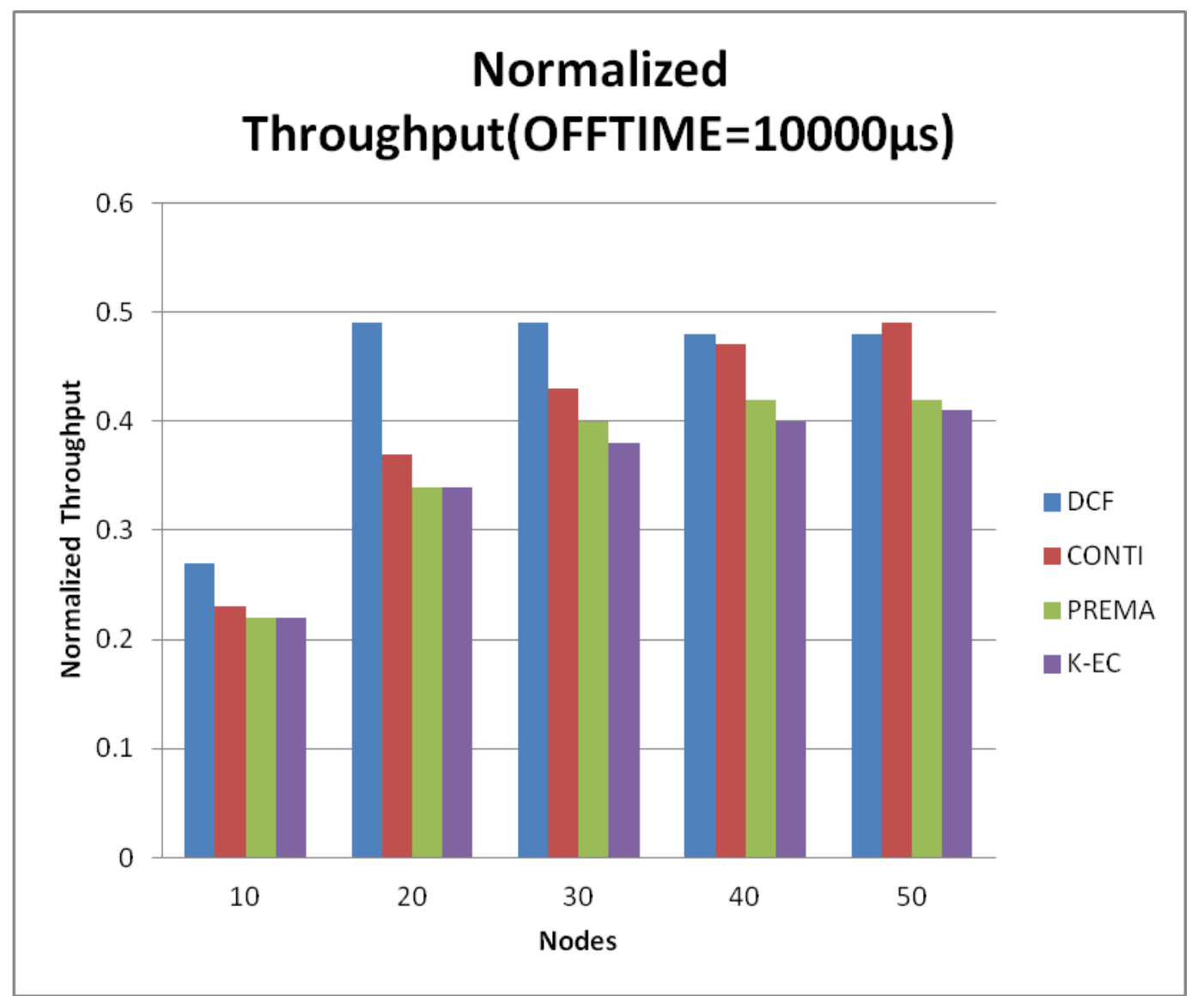

Fig 5.21: Bar chart for comparison of throughput for 10, 20, 30, 40 and 50 nodes for DCF, CONTI, PREMA and k-EC with mean offtime $=10000 \mu \mathrm{s}$

Almost up to 40 nodes, DCF has the highest throughput. However, from 40 nodes onwards the throughput of DCF decreases and we see an increase in the throughput of CONTI, PREMA and $\mathrm{k}-\mathrm{EC}$. When the number of nodes is 50, CONTI gives the best throughput result.

(c) Delay 


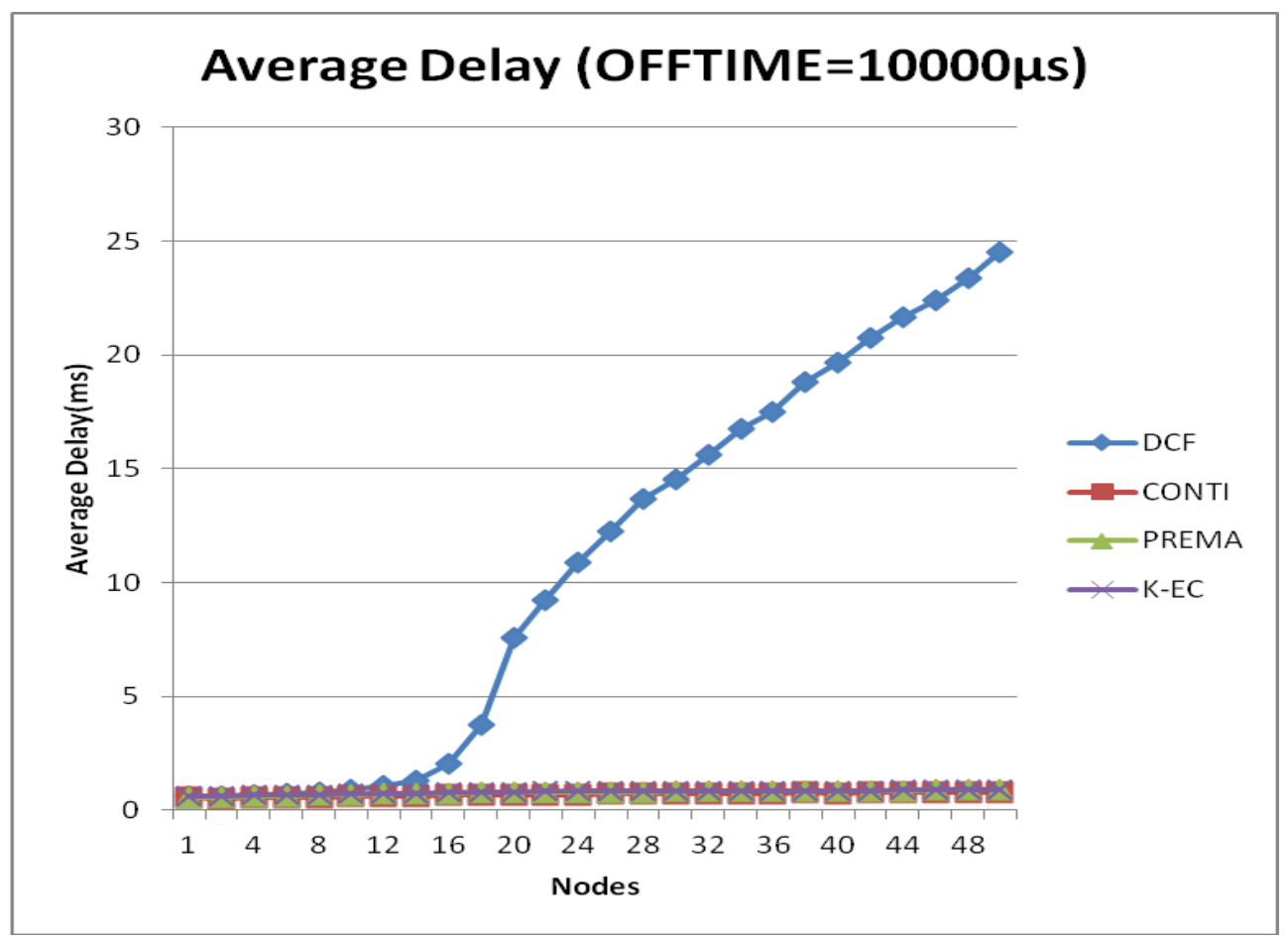

Fig 5.22: Plot for average delay with mean offtime $=10000 \mu$ s for DCF, CONTI, PREMA and kEC from 1 to 50 nodes

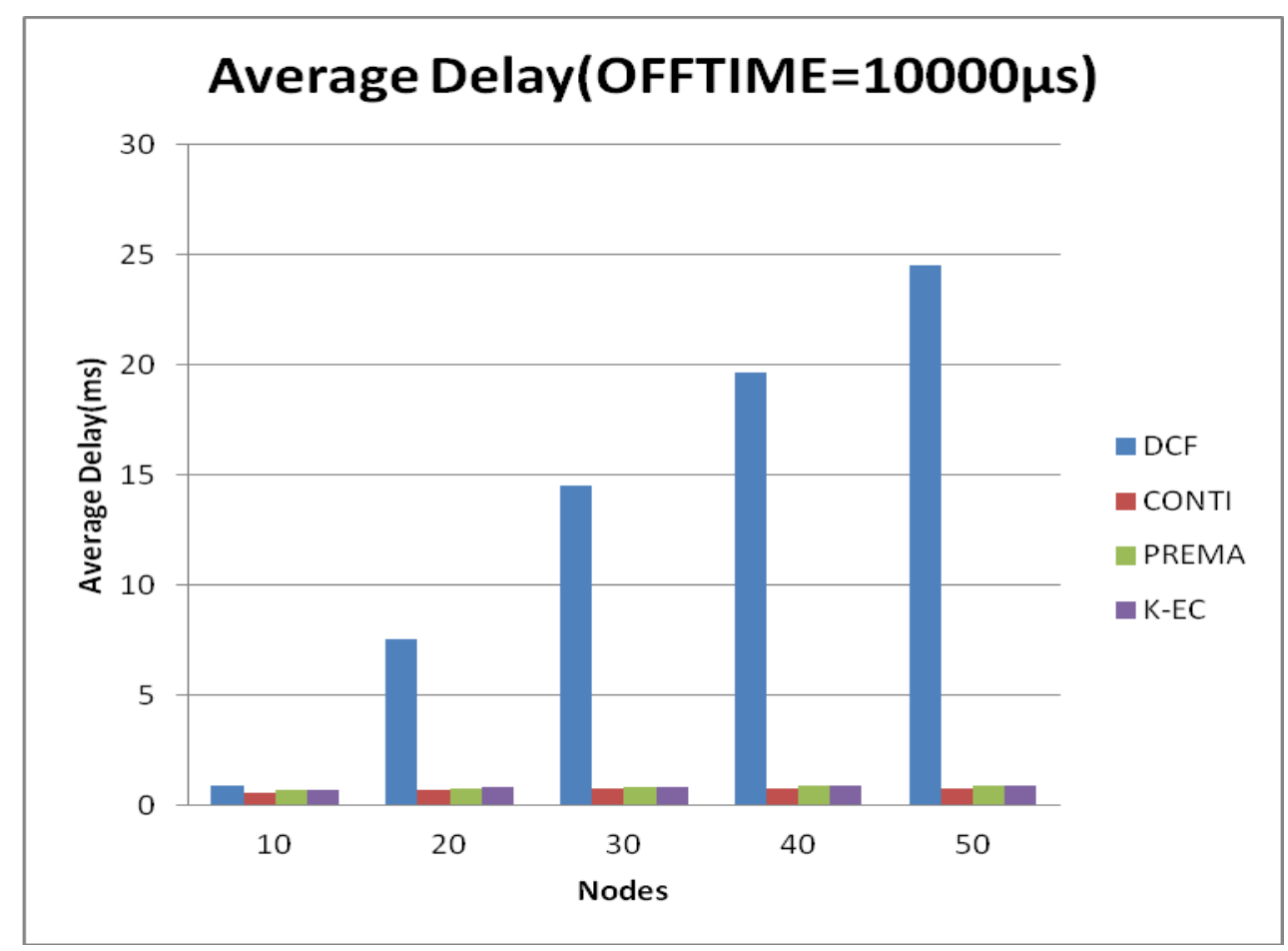

Fig 5.23: Bar chart for comparison of average delay for 10, 20, 30, 40 and 50 nodes for DCF, CONTI, PREMA and k-EC with mean offtime $=10000 \mu \mathrm{s}$ 
As the number of nodes increases, the delay for DCF is ever increasing. On the other hand, the delay for CONTI, PREMA and k-EC is relatively very low and thus these three schemes are better in terms of delay.

(d) Average time wasted in contention

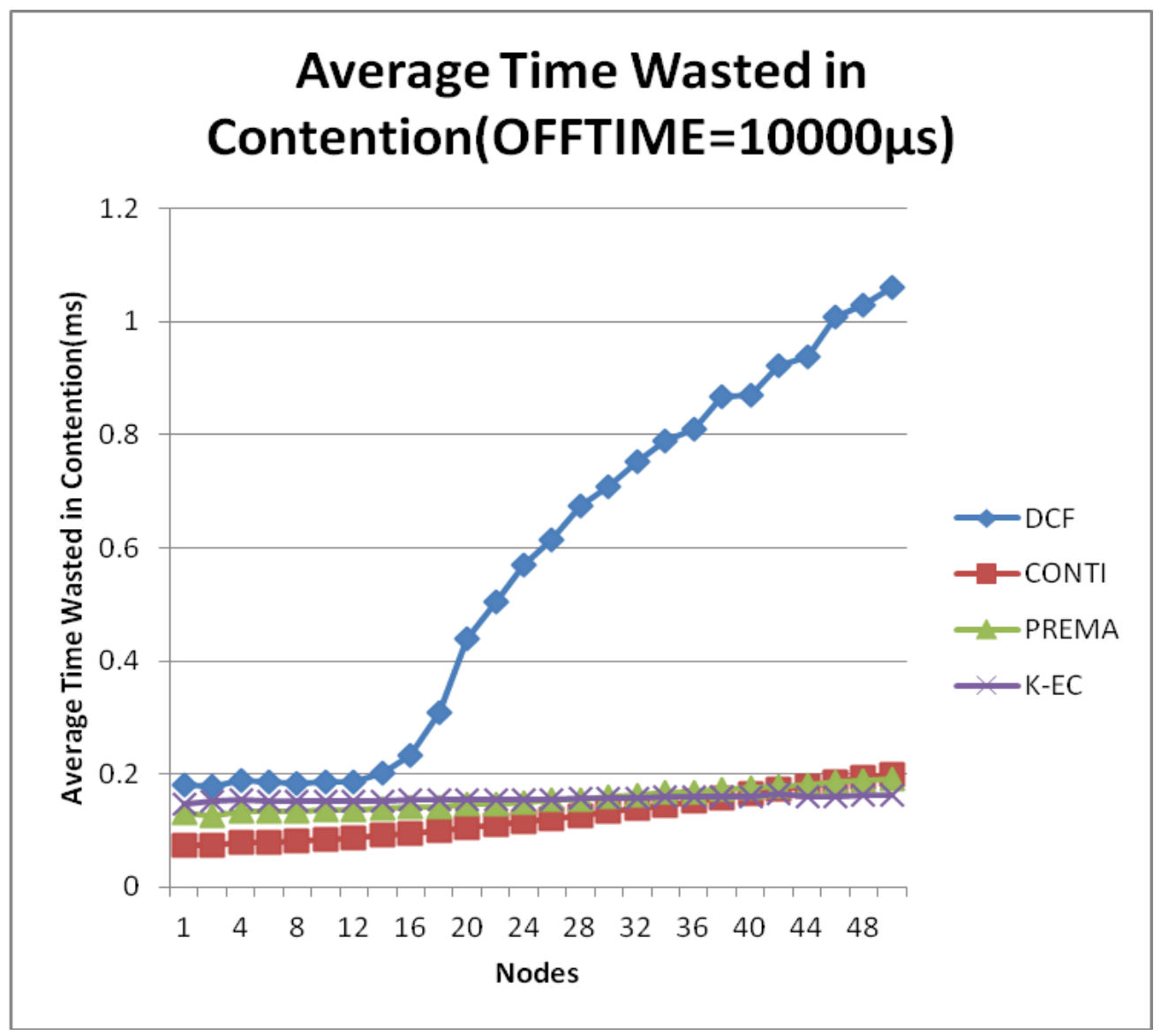

Fig 5.24: Plot for average time wasted in contention with mean offtime $=10000 \mu$ s for DCF, CONTI, PREMA and k-EC from 1 to 50 nodes 


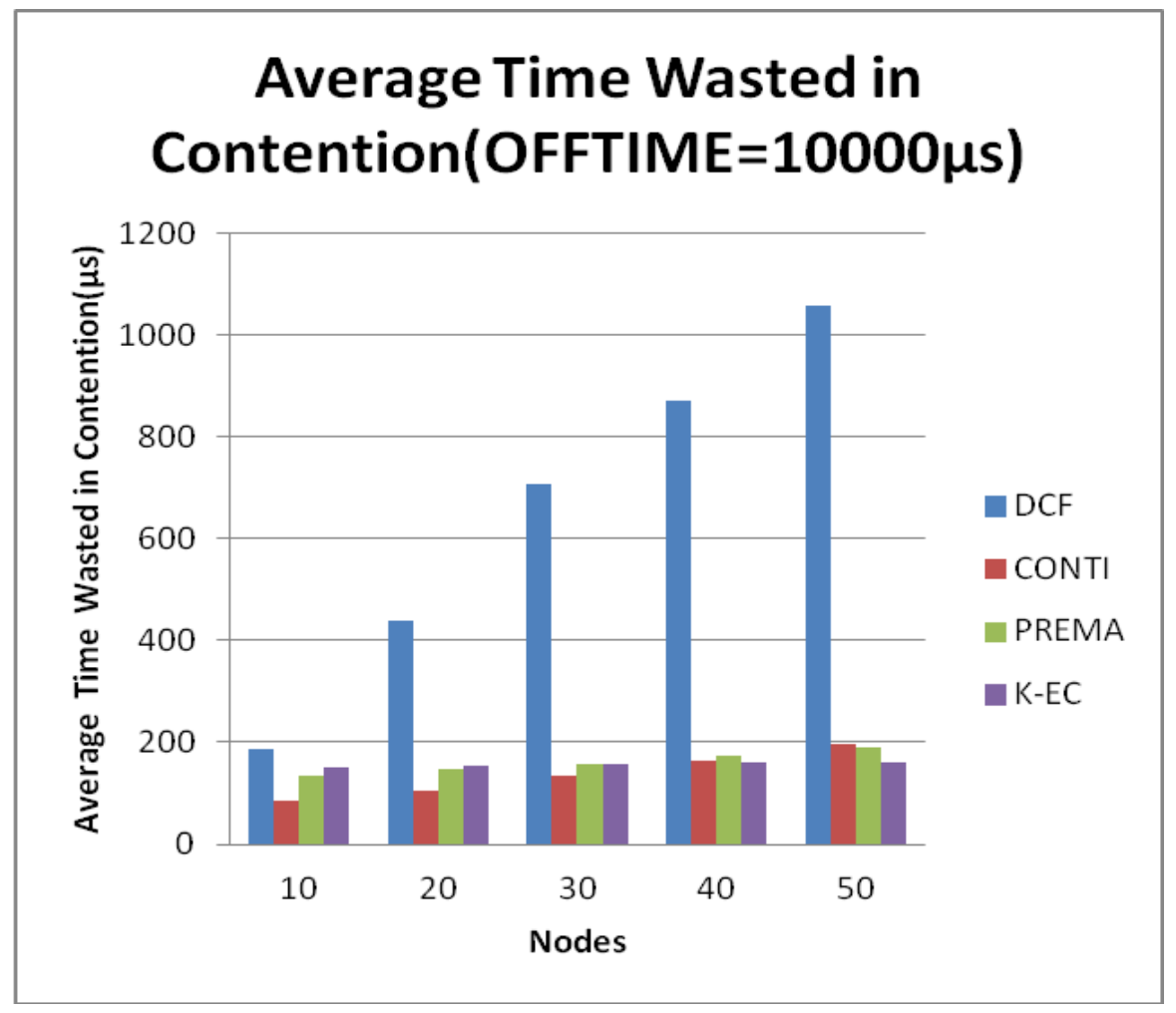

Fig 5.25: Bar chart for average time wasted in contention for 10, 20, 30, 40 and 50 nodes for DCF, CONTI, PREMA and k-EC with mean offtime $=10000 \mu \mathrm{s}$

DCF wastes the maximum amount of time in contention. For only a few nodes, CONTI gives a good performance in terms of time wasted in contention. When the number of nodes is larger, $\mathrm{k}$ EC takes lesser time.

(e) Average queuing delay 


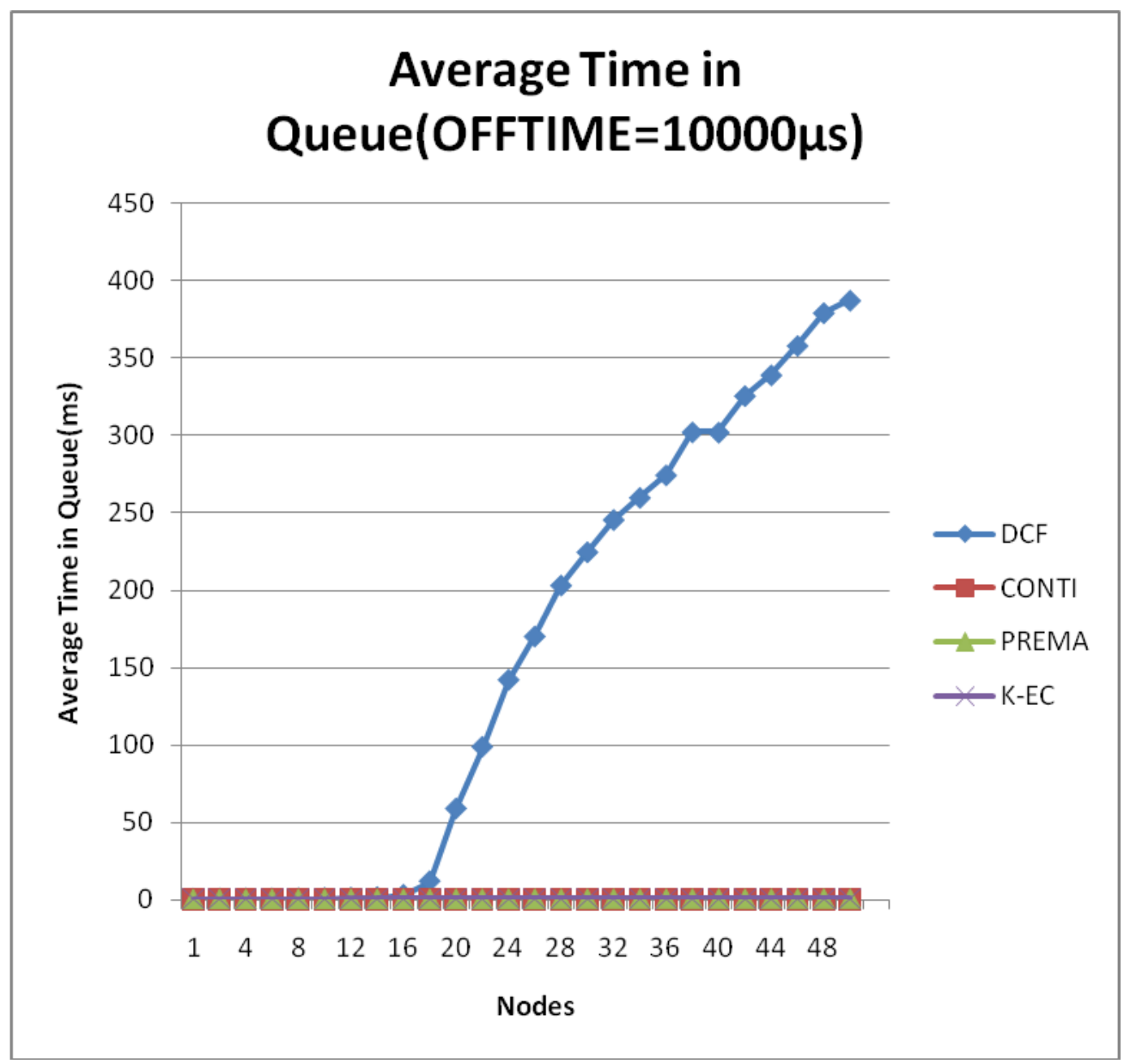

Fig 5.26: Plot for queuing delay with mean offtime $=10000 \mu$ s for DCF, CONTI, PREMA and kEC from 1 to 50 nodes 

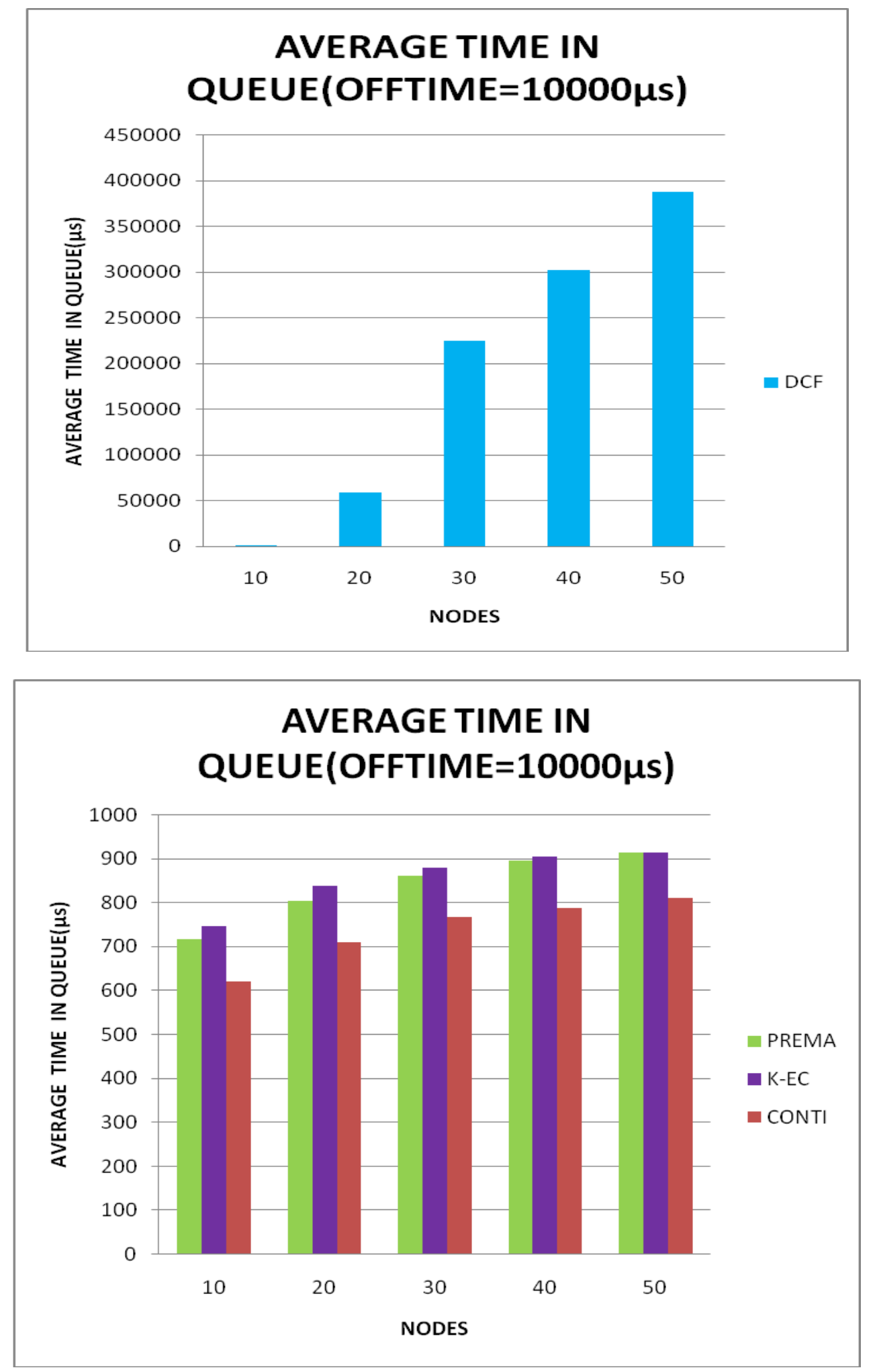

Fig 5.27: Bar chart for queuing delay for 10, 20, 30, 40 and 50 nodes for DCF, CONTI, PREMA and $\mathrm{k}-\mathrm{EC}$ with mean offtime $=10000 \mu \mathrm{s}$ 
The time spent by packets in queue in the DCF scheme is very high compared to the other schemes. CONTI shows the best performance.

\subsection{Summary of observations}

The first set of results where we used the parameter of $100 \mu$ s offtime was to verify that our proposed unsaturated model behaves similarly to the saturated model with the assumption that a very small offtime is close to zero. The remaining two sets of results with parameters $1000 \mu \mathrm{s}$ and $10000 \mu \mathrm{s}$ offtime were to check if the results of the unsaturated models were consistent with respect to parameter changes. In our findings we observe that we get the expected results with just a few deviations.

Across all scenarios, we find that CONTI outperforms the remaining contention schemes in terms of throughput, collision and delay. CONTI performs worst when it comes to average time spent in contention and this eventually hampers a potentially greater throughput that can be achieved with this scheme had it not been for the time wasted in contention. Although k-EC outperforms CONTI in terms of contention, average delay and time wasted in contention its throughput, which is a major contributing factor to the success of a scheme, is very poor. PREMA performs similarly to k-EC except k-EC is better than PREMA. As expected DCF performs poorly on all accounts except for its throughput and time wasted in contention compared to k-EC and PREMA. 


\section{[REFERENCES]}

[1] IEEE Std 802.11, Part 11: Wireless LAN Medium Access Control (MAC) and Physical Layer (PHY) Specifications, IEEE, 1999.

[2] G. Bianchi, "Performance Analysis of the IEEE 802.11 Distributed Coordination Function," IEEE J. Selected Areas in Comm., vol. 18, no. 3, pp. 535-547, Mar. 2000.

[3] E. Ziouva and T. Antonakopoulos, "CSMA/CA Performance under High Traffic Conditions: Throughput and Delay Analysis," Computer Comm., vol. 25, no. 3, pp. 313-321, 2002.

[4] A. Kumar, E. Altman, D. Miorandi, and M. Goyal, "New Insights from a Fixed Point Analysis of Single Cell IEEE 802.11 WLANs,” Proc. IEEE INFOCOM, 2005.

[5] M. Carvalho and J. Garcia-Luna-Aceves, "Delay Analysis of IEEE 802.11 in Single-Hop Networks," Proc. 11th IEEE Int'l Conf. Network Protocols (ICNP), 2003.

ABICHAR AND CHANG: A MEDIUM ACCESS CONTROL SCHEME FOR WIRELESS LANS WITH CONSTANT-TIME CONTENTION 203

Fig. 8. Fairness versus srame size. Fig. 9. Fairness versus number of stations.

[6] N.H. Vaidya, P. Bahl, and S. Gupta, "Distributed Fair Scheduling in a Wireless LAN," Proc. ACM MobiCom, Aug. 2000.

[7] P. Jacquet, P. Minet, P. Muhlethaler, and N. Rivierre, "Priority and Collision Detection with Active Signaling - The Channel Access Mechanism of HIPERLAN," Wireless Personal Comm., vol. 4, no. 1, pp. 11-26, Jan. 1997.

[8] J.L. Sobrinho and A.S. Krishnakumar, "Quality-of-Service in Ad Hoc Carrier Sense Multiple Access Wireless Networks," IEEE J. Selected Areas in Comm., vol. 17, no. 8, pp. 1353-1368, Aug. 1999.

[9] G. Wikstrand, T. Nilsson, and M. Dougherty, "Prioritized Repeated Eliminations Multiple Access: A Novel Protocol for Wireless Networks," Proc. IEEE INFOCOM, pp. 1561-1569, Apr. 2008.

[10] B. Zhou, A. Marshall, and T.-H. Lee, "A k-Round Elimination Contention Scheme for WLANs,” IEEE Trans. Mobile Computing, vol. 6, no. 11, pp. 1230-1244, Nov. 2007.

[11] Z. Abichar and J.M. Chang, "CONTI: Constant-Time Contention Resolution for WLAN Access,” Proc. Fourth Int'1 IFIP-TC6 Networking Conf., 2005.

[12] M. Heusse, F. Rousseau, R. Guillier, and A. Duda, "Idle Sense: An Optimal Access Method for High Throughput and Fairness in Rate Diverse Wireless LANs," Proc. ACM SIGCOMM, 2005. 
[13] Y. Grunenberger, M. Heusse, F. Rousseau, and A. Duda, "Experience with an Implementation of the Idle Sense Wireless Access Method," Proc. ACM Int'l Conf. Emerging Networking Experiments and Technologies (CoNEXT '07), pp. 1-12, Dec. 2007.

[14] H. Wu, A. Utgikar, and N. Tzeng, "SYN-MAC: A Distributed Medium Access Control Protocol for Synchronized Wireless Networks," Mobile Networks and Applcations, vol. 10, no. 5, pp. 627-637, Oct. 2005.

[15] T. You, C. Yeh, and H. Hassanein, "A New Class of Collision Prevention MAC Protocols for Wireless Ad Hoc Networks,” Proc. IEEE Int'1 Conf. Comm. (ICC), 2003.

[16] C. Yeh and T. You, "A QoS MAC Protocol for Differentiated Service in Mobile Ad Hoc Networks,” Proc. IEEE Int’l Conf. Parallel Processing (ICPP), 2003.

[17] J. Stine, G. DeVeciana, K. Grace, and R. Durst, "Orchestrating Spatial Reuse in Wireless Ad Hoc Networks Using Synchronous Collision Resolution (SCR)," J. Interconnection Networks, vol. 3, nos. 3/4, pp. 167-195, Sept.-Dec. 2002.

[18] J. Galtier, "Analysis and Optimization of MAC with Constant Size Congestion Window for WLAN,” Proc. Second Int’l Conf. Systems and Networks Comm. (ICSNC '07), 2007.

[19] L. Bononi, M. Conti, and E. Gregori, "Runtime Optimization of IEEE 802.11 Wireless LANs Performance," IEEE Trans. Parallel and Distributed Systems, vol. 15, no. 1, pp. 66-80, Jan. 2004.

[20] K.C. Tay, K. Jamieson, and H. Balakrishnan, "Collision-Minimizing CSMA and Its Applications to Wireless Sensor Networks," IEEE J. Selected Areas in Comm., vol. 22, no. 6, pp. 1048-1057, Aug. 2004.

[21] Q. Ni, I. Aad, C. Barakat, and T. Turletti, "Modeling and Analysis of Slow CW Decrease for IEEE 802.11 WLAN," Proc. 14th IEEE Int'l Symp. Personal, Indoor and Mobile Radio Comm. (PIMRC), 2003.

[22] H. Wu, S. Cheng, Y. Peng, K. Long, and J. Ma, "IEEE 802.11 Distributed Coordination Function (DCF): Analysis and Enhancement," Proc. IEEE Int'l Conf. Comm. (ICC), 2002.

[23] F. Cali, M. Conti, and E. Gregori, "Dynamic Tuning of the IEEE 802.11 Protocol to Achieve a Theoretical Throughput Limit," IEEE/ACM Trans. Networking, vol. 6, no. 8, pp. 785799, Dec. 2000.

[24] K. Jamieson, H. Balakrishnan, and Y.C. Tay, "Sift: A MAC Protocol for Event-Driven Wireless Sensor Networks," Proc. Third European Workshop Wireless Sensor Networks (EWSN), 2006. 
[25] T. Cormen, C. Leiserson, R. Rivest, and C. Stein, Introduction to Algorithms, second ed. MIT Press, 2001.

[26] IEEE Std 802.11b, Part 11: Wireless LAN Medium Access Control (MAC) and Physical Layer (PHY) Specifications: Higher-Speed Physical Layer Extension in the $2.4 \mathrm{GHz}$ Band, IEEE, 1999.

[27] Z. Abichar, J.M. Chang, and D. Qiao, “Group-Based Medium Access for Next-Generation Wireless LANs," Proc. Int'l Symp. World of Wireless, Mobile and Multimedia Networks (WOWMOM '06), pp. 35-41, 2006.

[28] R. Jain, The Art of Computer Systems Performance Analysis. John Wiley \& Sons, 1991.

[29] C.E. Koksal, H. Kassab, and H. Balakrishnan, "An Analysis of Short-Term Fairness in Wireless Media Access Protocols,” Proc. ACM SIGMETRICS, June 2000. 